\title{
Übergänge zur Schriftlichkeit. \\ Zu wechselnden Profilen dialektaler Literalität \\ am Beispiel des Niederdeutschen
}

Robert Langhanke (Flensburg)

\begin{abstract}
Different types of Low German literacy create varieties in addition to spoken Low German. Their function differs according to the change of language use. By setting the focus on dialectal literature it becomes clear, which concepts of Low German literature became influential since the $19^{\text {th }}$ century.

In the recent situation, new perspectives for Low German and its literature can be found in the field of planned language acquisition for example at school. Therefor written forms of Low German become much more important than usually thought of by looking at the ideas of language policy and the development at schools in Northern Germany.
\end{abstract}

\section{$1 \quad$ Einleitung}

Eine kulturell gesteuerte Reaktion auf den Rückgang gesprochener Mundarten ist deren geregelte Übernahme in schriftliche Ausdrucksformen. Seit Beginn des 19. Jahrhunderts wurden zahlreiche Mundartliteraturen etabliert, die durch vergleichbare sprachliche Anforderungen bei der Verschriftlichung und umfassende Domäneneinschränkungen aufgrund ihrer Existenzform neben einer Standardsprache geeint werden. Unter diesen Mundartliteraturen nimmt die Sammelgruppe der niederdeutschen Literaturen wegen ihrer starken internen Traditionsbildung und dem weit entwickelten Konstrukt einer Dichotomie von niederdeutscher und hochdeutscher Sprache und Literatur eine Sonderstellung ein. ${ }^{1}$

Am Beispiel der umfassend ausgebauten, zahlreiche Textsorten betreffenden neuniederdeutschen Schriftlichkeit werden historische und rezente Bedingungen dialektaler Literalität geprüft, indem Konzepte für die Herausforderung, ein eigenständiges, standardabweichendes System gesprochener Sprache in ein etabliertes, standardgeprägtes Zeichensystem geschriebener Sprache zu übertragen, kontrastiv betrachtet und mit den möglichen sprachlichen Kompetenzen der Produzenten und Rezipienten abgeglichen werden, soweit Einschätzungen geboten scheinen. Die in den Blick genommene Schriftlichkeitstradition erweist sich als umfassend ausgebaut, da seit ihrer Reetablierung im Verlauf des 19. Jahrhunderts kontinuierlich

1 Den beiden Gutachterinnen/Gutachtern sei für die genaue Lektüre und die hilfreichen Hinweise sehr gedankt! Linguistik online 85, 6/17 - http://dx.doi.org/10.13092/lo.85.4083

CC by 3.0 
versucht wurde, sie für verschiedene Textsorten auch des Gebrauchsschrifttums zu qualifizieren, wobei der Primat jedoch immer der Nische einer neuen Literatursprache galt.

Neben Fragen zur Grammatik, Stilistik und Orthographie in diesem Sonderfall des Übergangs von Mündlichkeit zur Schriftlichkeit muss die Diskussion der wechselnden Motivation und Funktion dialektaler Literalität stehen. Dieser letzteren Fragestellung gilt das besondere Augenmerk dieses Beitrags, der keine umfassende Korpusanalyse neuniederdeutscher literarischer Texte bieten kann, obgleich diese immer wieder angemahnt wurde (cf. Langhanke 2011, 119, Anm. 14 und 120, Anm. 18: „Flensburger Mundartliteraturkorpus“) und vom Autor als Aufgabe erkannt ist. Beispiele grammatischen, stilistischen und orthographischen Transfers werden sich gleichwohl anbringen lassen. Die Frage nach der Motivation kann bereits als besser aufgearbeitet und auf einer Quellenlage metasprachlicher Äußerungen, derer die niederdeutsche Literaturtradition nicht allzu reich ist, angegangen werden: Ursprünglich sollten die Verschriftlichungen ältere mundartliche Formen bewahren. Gegenwärtig führt die Annäherung gesprochener Dialekte an standardsprachliche Strukturen jedoch zu sprachlichem Wandel, der auch in dialektaler Schriftlichkeit gespiegelt wird. Diese basiert nur in selten gewordenen Fällen auf bestehender Schriftlichkeit als Leitvarietät, sondern in der Regel auf einer gesprochensprachlichen Kompetenz des Autors.

Zahlreiche der einzelnen „Literaturmundarten“ (Haas 1983: 1642) repräsentieren dabei dennoch weiterhin ihre sprechsprachlichen Ausgangsmundarten und können als ein schriftsprachliches Vorbild auf Vermittlungsprozesse und weitere Verschriftlichungen einwirken. Daher erhebt sich gegenwärtig die Frage, inwieweit diese Verschriftlichungen umgekehrt bei einem Übergang zu neuer Mündlichkeit Impulse zu sprechsprachlichem Gebrauch der Mundarten geben - wegen der erst begonnenen institutionellen Vermittlung liegen hierzu noch keine empirischen Daten vor.

Die Übergänge zur Schriftlichkeit sind in älteren und neueren Texten durch verschiedene Strategien zur Abbildung von Mündlichkeit in den schriftlichen Umsetzungen von Dialekten als ursprünglich allein gesprochenen Sprachformen geprägt. Übernehmen jüngere Verschriftlichungen bewusst archaisierende dialektale Formen, oder bilden sie rezente dialektale Mündlichkeit ab, und welches Verfahren ist für welche Zielsetzung wünschenswert (cf. die Diskussion bei Elmentaler/Borchert 2012; dazu anders Wirrer 2014)? Lassen sich dialektliteratursprachliche Traditionsbildungen neben den Einflüssen der Standardschriftsprache und der rezenten (dialektalen) Mündlichkeit auf dialektale Literatursprache ausmachen? Diese Fragen werden mit einer Perspektive auf die Motivationen und Zielsetzungen der Dialektverschriftlichungen diskutiert, jedoch zunächst nur im Ansatz sprachlich exemplifiziert.

\section{$2 \quad$ Formen dialektaler Literalität}

\subsection{Mundartliche Schriftlichkeit}

Der mediale Übergang von gesprochener Mundart in geregelte Schriftlichkeit am Beispiel der niederdeutschen Dialekte ab dem 19. Jahrhundert markiert einen entscheidenden sprachgeschichtlichen Entwicklungsschritt und wirft vornehmlich Fragen auf, die zum einen die externe Motivation und zum anderen die interne Ausgestaltung betreffen (cf. zur Qualifizierung für die Sprachgeschichte Langhanke 2015b). Die Emanzipation einer Dialektverschriftlichung 
von der umgebenden standardsprachlichen Schriftlichkeit wird zur besonderen Herausforderung.

Die folgenden Überlegungen werden am Beispiel des Niederdeutschen entwickelt, da bei aller Vergleichbarkeit generelle Aussagen über Mundartliteratur nur eingeschränkt möglich sind und stets an eine konkrete Region und deren regionalsprachlichen Verhältnisse ${ }^{2}$ zurückgebunden werden müssen. Besondere Bedingungen gelten für den alemannisch-schweizerischen und den niederdeutschen Raum, die nur bedingt auf andere Mundarträume und ihre mundartliche Literaturproduktion unter dem Dach der hochdeutschen Standardsprache zu beziehen sind. Diese Sonderstellung ist qualitativ und quantitativ motiviert.

Vornehmlich Klaus Groth (1819-1899) und Fritz Reuter (1810-1874) stehen im Folgenden für den Aufschwung neuniederdeutscher Schriftlichkeit nach der Mitte des 19. Jahrhunderts. ${ }^{3}$

Am 11. September 1852 schrieb der niederdeutsche Lyriker Klaus Groth an den Freund Ernst Griebel: „Man hat mit sonderbaren Schwierigkeiten zu kämpfen, die eben nur der Produzierende gewahr wird. Schon das Erhaschen des mundartlich Richtigen ist merkwürdig schwer und selbst angreifend. Man gerät zuerst immer wieder in den gebildeten Schriftton." (Groth 1852/1938: 302) Damit thematisiert Groth die hochdeutsche Standardschriftlichkeit als die ungewollte, aber doch stets präsente Bezugsgröße einer dialektalen Literalität. Am Beginn des 21. Jahrhunderts hat dialektale Schriftlichkeit viele Erscheinungsformen. Dialektbasierte Schriftlichkeit begegnet auf mehreren Existenzebenen in unterschiedlicher Ausprägung, und entsprechend vielschichtig sind an sie gerichtete Erkenntnisinteressen. Wenigstens acht unterschiedliche Existenzformen lassen sich differenzieren.

Erstens gilt: Historische Schreibsprachen sind dialektbasiert, also erkennbar raumorientiert. In privaten Textsorten setzte sich ihre Tradition lange fort (cf. zu den frühneuzeitlichen Schreibsprachen Mihm 2007, Langhanke 2012b, Peters 2017/im Druck; Denkler 2006 zu späteren Sterbfallinventaren), und unter Berücksichtigung der historischen Entwicklungsfolge ist auch die moderne Standardschriftsprache in vielen Details fortgesetzt dialektbasiert, auch wenn diese Basis überformt ist und unkenntlich wurde. - Zweitens gilt: Dialekte wurden nach der Etablierung einer hochdeutschen Standardschriftsprache in einem kulturhistorisch eingrenzbaren Prozess als Sprache von Literatur wieder zu neuen Schreibsprachen (Mundartliteratur), die als „Literaturmundarten“ (Haas 1983: 1642 und öfter; cf. zudem Langhanke 2010 und neuerdings Herrmann/Siebenhaar 2015: 52-58) bezeichnet werden. Die Frage, ob ein neutralerer Begriff „Literatursprache“ dem auch in sich widersprüchlichen Begriff „Literaturmundart" vorzuziehen wäre, muss verneint werden, da der Anspruch eines Terminus für die meisten konkreten Existenzformen zu weitreichend ist: Alle Bedingungen von Literatursprache sind bei weitem nicht erfüllt, auch wenn sie bisweilen erwünscht wurden. - Drittens gilt, dass konkrete Realisierungen standardisierter Schriftsprache unter erkennbarem dialektalen Einfluss stehen können. - Viertens gilt, dass es im Verborgenen eine dialektale Alltagsschriftlichkeit gibt (so zum Beispiel Notiz- und Einkaufszettel auf Niederdeutsch) die wiederum

\footnotetext{
2 Der Begriff „Regionalsprache“ wird im Sinne von Schmidt/Herrgen 2011 als „vernetztes Gesamt an Varietäten und Sprechlagen“" unterhalb der „nationalen Oralisierungsnorm der Standardvarietät" (66) verstanden.

3 In der niederdeutschen Philologie, nicht jedoch in der hochdeutschen Sprach- oder Literaturwissenschaft, ist beiden Autoren eine breitere Forschungstradition gewidmet, die jedoch nicht immer einem streng wissenschaftlichen Diskurs folgen mochte; cf. jedoch einschlägig Batt 1958, 1967 und neuerdings Langhanke 2015, 2016.
} 
private Textsorten betrifft. ${ }^{4}$ - Fünftens gilt, dass verschriftlichte „Literaturmundarten“ (siehe oben zur Verteidigung dieses etablierten Terminus) bewusst für private oder halbprivate Schriftlichkeit verwendet und gestaltet werden (niederdeutsche Korrespondenz). - Sechstens gilt, dass Dialekt in den modernen elektronischen Kommunikationsmedien und -netzwerken als konzeptionell mündliche schriftliche Ausdrucksform Anwendung findet (cf. Tophinke 2008; Wyss/Ziegler 2008; Fredsted 2015; Reersheemius 2015b): Die Dialektverschriftlichungen in den neuen Medien zeugen von ausgeprägter Sprachbewusstheit und kreativem Umgang mit schriftsprachlichen Zeichen. - Siebtens gilt, dass geschriebene dialektale Wörter und Namen den öffentlichen Raum mitgestalten, zum Beispiel auf ausgewählten Schildern (cf. Reershemius 2015a). Werbeabsichten und sprachpolitische Weisungen geben die entsprechenden Impulse. - Achtens gilt schließlich, dass es eine dialektologisch motivierte Tradition schriftlicher Dialektübertragungen an der Schnittstelle von bewusst fixierter Mündlichkeit und Mundartliteratur gibt (cf. Haas 1983; Langhanke 2009; siehe auch Kleiner 2006). Das bekannteste Beispiel sind die schriftlichen Übertragungen der Wenkersätze, derer sich auch gestandene Mundartdichter des ausgehenden 19. Jahrhunderts befleißigten - mit gutem dialektologischen Erfolg (cf. Langhanke 2011a, 2011b).

\subsection{Mundartliterarische Schriftlichkeit}

Die soeben an zweiter Stelle erfasste Mundartliteratur steht im Folgenden im Fokus. Sie nahm ihren Ausgangspunkt Ende des 18. Jahrhunderts bei der Notierung und Bearbeitung von sogenannter Volksüberlieferung - Sagen, Lieder, Sprüche, Rätsel, Märchen (cf. Haas 1983; Jaeger 1964). Da die Sammler sowohl bearbeiteten als auch verschriftlichten, war der Schritt zu einer bewussten, ästhetisch-philologisch und akademisch motivierten Verschriftlichung eines Dialekts vollzogen. Im Rahmen eines kulturhistorisch begründbaren Literaturprogramms entstand bald eigenständige Mundartliteratur, die sich zunächst an den Formen der gesammelten Überlieferungen orientierte. Diese um 1800 begründete Tradition bewusst gesteuerter und kreativ erzeugter Dialektverschriftlichungen mit programmatischer Tendenz und dialektologischem Feingefühl im Sinne einer genauen Abbildung der zugrundeliegenden Mundart besteht bis heute. Kreative Freiheit hat ihre Grenze dort, wo der grammatische Eingriff in die Mundart beginnt. Von dieser Einengung können manche Beispiele moderner Mundartliteratur, die mit bewusster sprachlicher Verfremdung arbeiten, ausgenommen werden, doch wird die Ebene des sprachlich-dialektal Möglichen in der Regel auch dort nicht verlassen, zumal die Autoren ihre Literatursprache kennen und entsprechend einschätzen und belasten können. ${ }^{5}$

Dialektbezogene Schriftlichkeit in den neuen Medien steht zu dieser Tradition in keiner Verbindung. Sie ist privater ausgerichtet als Mundartliteratur, die eine künstlerische Veräußerli-

\footnotetext{
4 Auch hier offenbart sich ein unerforschtes Terrain, Sammlungen entsprechenden Materials stehen am Beginn, sind sie doch der Inbegriff eines Gebrauch- und Wegwerf-Schriftlichkeit, die niemanden außer dem Schreiber interessieren sollte, die vielleicht auch ansonsten niemand lesen könnte. Doch zum Beispiel Flensburger Studierende konnten niederdeutsche Einkaufszettel ihrer Großeltern problemlos einbringen.

5 In den 1960er bis in die 1990er Jahren etablierten Autoren wie Oswald Andrae, Siegfried Kessemeier, Norbert Johannimloh, Greta Schoon und zudem sehr eigenständig Waltrud Bruhn sowie andere Dichter einen Anschluss niederdeutscher Lyrik an experimentelle, nicht traditionalistische Formen (cf. Stiftung F.V.S. zu Hamburg 1989; Goltz 1996, 1998; Langhanke 2010). Breitere Wahrnehmung konnte sich jedoch nicht einstellen.
} 
chung dialektalen Materials bedeutet. ${ }^{6}$ Während Mundartliteratur zumindest Mikrostandardisierungen (in Bezug auf einzelne Texte oder einzelne Autoren) einfordert, ist dialektale Schriftlichkeit in den neuen Medien von entsprechenden Konzepten weit entfernt und kann in jeder Umsetzung neu entstehen; auch textinterne Regeln bestehen nicht.

Seit der Etablierung von Mundartliteratur nach 1800 ist im niederdeutschen Raum wiederholt der programmatische Anspruch formuliert worden, Dialektschriftlichkeit in weitere Domänen vordringen zu lassen, um die Standardschriftsprache zu ergänzen oder gar abzulösen, oder um lediglich Textsorten auch außerhalb literarischer Formen im engeren Sinne in den Blick zu nehmen (cf. das Projekt Rüschenschmidt 2006). Die Schnittstelle von Literatur- und Gebrauchsschriftlichkeit wurde und wird jedoch kaum genutzt, auch wenn in der Europäischen Charta der Regional- oder Minderheitensprachen ein Recht auf Schriftsprachgebrauch festgeschrieben ist. Dieses ist im Niederdeutschen allein über die mundartliterarische Tradition belebt, denn lebenspraktische Notwendigkeiten für einen niederdeutschen Schriftsprachgebrauch bestehen nicht.

Mundartliteratur ist ein medialer Übergangsraum des Dialektgebrauchs. Spracheinstellungen und Sprachwissen können von ihr mitbestimmt sein. Wenn Mundartliteratur als produktiv, stagnierend oder abklingend erfasst wird, wird zugleich eine Aussage über den Sprachstand einer gesamten Mundartregion getroffen, so dass auch diese spezifische Ausprägung des Dialektalen als Gradmesser für den Sprachstand dienen kann. Traditionell wird Mundartliteratur mit der (zumindest erwünschten) Konsolidierung von Dialekten verbunden, doch hat Dialektschriftlichkeit diesen Anspruch nur eingeschränkt erfüllen können. Die neue Sichtbarkeit und die Möglichkeit eines Bezugs auf die zuvor nur am Ort ihrer Sprecher flüchtig greifbare und nun schriftlich konservierte dialektale Sprache erzielte neue Wertschätzung, konnte aber keine sprechsprachlichen Domänen zurückgewinnen lassen. Dennoch repräsentiert Mundartliteratur bei aller sprachlichen und inhaltlichen Kritik an ihren konkreten Ausprägungen, die sich vornehmlich über ihre weitgehende Nichtbeachtung im standardsprachlichen Literaturbetrieb erleben lässt, seit über 200 Jahren dialektale Literalität mit einem Wirkungsgrad für die Existenz der mundartlichen Sprachformen, der ihren Beachtungsgrad im allgemeinen kulturellen Leben der jeweiligen Sprachräume weit übersteigt, wie zum Beispiel über die zumindest temporären Ausdifferenzierungen innerhalb einer neueren niederdeutschen Kulturszene gezeigt werden kann. Unter den acht genannten aktuellen Feldern dialektaler Schriftlichkeit bildet die Mundartliteratur die am stärksten vertretene und auch die geregeltste Erscheinungsform, an die im Verlauf ihrer Entwicklungsgeschichte unterschiedliche Zielsetzungen herangetragen wurden:

Zum einen, auch wegen der Auftretenshäufigkeit, ist die Herausarbeitung von grammatischer Information aus mundartliterarischen Texten, deren Schriftsprachlichkeit regionale historische Sprachstände konserviert (cf. Jørgensen 1934, Langhanke 2011a, 20011b), ein lang etabliertes Verfahren. Traditionell trat dialektale Schriftlichkeit als Quelle für dialektologische Untersuchungen auf (cf. Jaeger 1964; Haas 1983; Langhanke 2009), bevor weitere, im engeren Sinne dialektologische Verfahren im 19. Jahrhundert entwickelt wurden (cf. Knoop 1982).

\footnotetext{
${ }^{6}$ Im Internet publizierte Mundartliteratur wird nicht als dialektbezogene Schriftlichkeit in den neuen Medien im engeren Sinne betrachtet, sondern als neue Präsentationsform der traditionellen literarischen Dialektverschriftlichungen bewertet.
} 
Gegenwärtig wird zum anderen die Vermittlung von Dialekten als Sprechsprache gefordert, wie Bemühungen um die Bewertung von Niederdeutsch als Fremdsprache im norddeutschen Schulunterricht zeigen (cf. Langhanke 2013; Goltz 2013). Die Funktion niederdeutscher Literalität im zugehörigen Sprachvermittlungsprozess muss dabei kritisch ausgelotet und schließlich an den Verfahren etablierter Fremdsprachdidaktiken orientiert werden (cf. Bendixen 2016; Wormuth 2015), um Lernerfolge erzielen zu können.

Diese beiden Perspektiven zeigen als widerstreitende Zielsetzungen zum einen die detaillierte Spiegelung raumbildender Merkmale bei Vermeidung von Sprachausgleich in der Mundartliteratur und zum anderen die Normierung von Sprache zur Unterstützung von Sprachlernprozessen und zur Ausprägung von Konventionen im Schulfach Niederdeutsch. Die Abbildung oder Vermeidung raumbildender Merkmale wird flankiert durch die Frage nach ihrem Auswahlkriterium - welche Merkmale sollte eine mundartliterarische Verschriftlichung mündlichkeitsnah abbilden, und welche Merkmale sollten im Zuge einer Normierung abgebaut werden? $\mathrm{Ob}$ in bestimmten mundartliterarischen Umsetzungen gesteuerte Regionalisierung von Schriftlichkeit stattfand oder ob diese mehrheitlich „naiv“ an Mündlichkeit anschloss, bleibt oftmals verdunkelt: Den Übergängen von der Mündlichkeit zur Schriftlichkeit liegen ästhetische, sprachlich-philologische, sprachpraktische oder kombinierte Konzepte zugrunde, die entweder die künstlerische Wirkung der Mundarttexte, die Dokumentation eines Dialekts sowie seinen Erhalt oder die Summe dieser Ziele in den Mittelpunkt stellten. Ob diese Konzepte bereits ursprünglich bestanden oder erst nachträglich erschlossen wurden, ist nur bedingt einschätzbar, da den meisten Mundarttexten keine theoretischen Überlegungen beigegeben sind, die sich zum Beispiel in Vor- und Nachworten der Erstausgaben einer Buchpublikation finden könnten. ${ }^{7}$ Die Rekonstruktion entsprechender Konzepte informiert über Spracheinstellungen und Einschätzungen zur Flexibilität einer Sprachform im MündlichkeitSchriftlichkeit-Transfer. Zugespitzt gilt „das Niederdeutsche ist eine Schriftsprache“ oder „das Niederdeutsche ist keine Schriftsprache.“ Zwischen diesen Polen bewegen sich graduell verschiedene Einschätzungen niederdeutscher Schriftsprache. Der Übergang einer niederdeutschen Mundart zur Schriftlichkeit ist zum einen als Verhochdeutschung beschreibbar, da Imitationen von Standardschriftlichkeit und -sprachlichkeit vorliegen, ${ }^{8}$ wenn die Mundart bewusst medial transferiert wird; so zum Beispiel in der Syntax, im Textaufbau und auch in stilistischer Hinsicht. Zum anderen liegt Bewahrung niederdeutscher Sprachstruktur vor, da grammatische Merkmale konstitutiv bleiben, die in der Mündlichkeit rückläufig sind: Schriftsprachlich-niederdeutsche Stilistik beruht auf dem Erhalt dieser Merkmale, als deren prominentes Beispiel die Verwendung von tun/doon-Periphrasen im Nebensatz herangezogen werden kann (cf. die Hinweise bei Elmentaler 2009a, 2009b, 2012 und insbesondere 2015 sowie zur Methodik Rohdenburg 1991, 2002). Alle Verschriftlichungen des Niederdeutschen in sei-

\footnotetext{
7 Die Tatsache, dass diese Reduzierung auf Buchliteratur insbesondere für Mundartliteraturen keineswegs angemessen ist, da viele Texte unterhalb dieser Schwelle existieren oder existierten, rückt mehr und mehr in das Bewusstsein; cf. zu den dafür einschlägigen Sammlungen und Arbeiten von Peter Bürger Langhanke 2015c.

8 Unter kulturgeschichtlicher Perspektive formuliert Menke als ein Kennzeichen der mittelniederdeutschen Literaturlandschaft (vornehmlich des 15. Jahrhunderts), dass „eine rezeptive Übersetzungsliteratur als repräsentative städtische Imitationskultur“ vorliegt (Menke 2000: 716). Dieses Stichwort der „Imitationskultur“ kann in einem sehr ausgeweiteten Sinne auch treffend auf die neuen schriftsprachlichen Bemühungen um das Niederdeutsche im 19. Jahrhundert angewandt werden und charakterisiert auch grundsätzlich hilfreich eine wichtige Facette im Verhältnis des Niederdeutschen zum Hochdeutschen, die im Grunde seit dem 8. Jahrhundert Gültigkeit besitzt.
}

ISSN 1615-3014 
nen verschiedenen Mundarträumen eint, dass sie bisher nicht in eine Standardform mündeten. Sie existieren im Spannungsfeld von sogenanntem Verfall und Innovation, Merkmalsabbau und Sprachausbau. Puristischen Vorstellungen stehen offene Konzepte gegenüber, deren Ausprägung von der philologischen-poetologischen Kompetenz und Motivation der Autoren sowie ihrer individuellen Kreativität abhängt. Als Schlüssel zur überregionalen SprachEinigung gelten seit Beginn neuniederdeutscher Verschriftlichung vornehmlich Orthographiekonzepte (cf. Hinsch 1956, 1983; Kellner 2002 und grundsätzlich Maas 1989), wobei Orthographie, grammatische Standardisierung und Sprachwandel vielfach miteinander verwechselt werden (cf. dazu auch Hinweise bei Kellner 2002: 240-244, bes. 244): Orthographie wandelt keine Grammatik, gilt jedoch traditionell und vornehmlich außerwissenschaftlich als Vehikel für eine konkrete Auseinandersetzung mit der niederdeutschen Standardisierungsdebatte.

Literatursprachliche Dialektverschriftlichungen ergeben „Literaturmundarten“, deren Entwicklung Gradmesser für den Stand der zugehörigen gesprochenen Dialekte ist, da Schriftlichkeit nur eine kurze Zeit jenseits der dialektalen Gesprochensprachlichkeit überleben kann (cf. Peters 1998/2012: 461). Ihren Ausgangspunkt haben die „Literaturmundarten“ in der Exotisierung und Musealisierung von Mundart, deren relative Ursprünglichkeit den Impuls gab, sie als Gegenstand von Dokumentation und Gestaltung zu begreifen. Ihre übergreifende Erläuterung gebietet Vorsicht, da bei aller Parallelität der Ausgangssituationen nur der Blick auf regionale Verhältnisse Strukturen erklären kann, wie die niederdeutschen, deutschschweizerischen und luxemburgischen Dialektliteraturen - die jeweils aus einer Gruppe von „Literaturmundarten" bestehen - beispielhaft verdeutlichen. Es haben sich unterschiedliche mundartliterarische Systeme etabliert, deren Textproduktion und Textrezeption jeweils spezifisch sind.

Die historische Dimension dialektaler Schriftlichkeit stützt die Frage nach den erwünschten Profilbildungen. $\mathrm{Ob}$ sich die Motive für die Verschriftlichung gewandelt haben und ob die schriftsprachlichen Formen Veränderungen und Perspektiven für den niederdeutschen Dialektverband bewirkten, lässt sich über einige Prinzipien eingrenzen:

Es gibt keine standardisierte schriftsprachliche Form, sondern niederdeutsche Literatursprache existiert als unendlich große Gruppe raumgebundener „Literaturmundarten“ mit regional unterschiedlichem Standardisierungsgrad. Die Entwicklung von Standardisierungen ist regional wiederholt begonnen worden, wobei die Orthographiediskussion die Stellvertreterrolle für Fragen zur sprachlichen Verfasstheit, Funktion und Wirkung der konkurrierenden Mundartliteraturen übernahm. Sie bot Raum für einen kritischen Diskurs über dialektale Literalität, der ansonsten kaum geführt wurde, denn die Grenzen inhaltlicher Gestaltung wurden zunächst akzeptiert und stilistische Kategorien kaum ausgebildet - mit der Folge, dass stilistische Wahlmöglichkeiten niederdeutscher „Literaturmundarten“ sehr begrenzt sind; so überwiegt inzwischen insbesondere eine mündlichkeitsnahe (Reihung kurzer Syntagmen) und humorbezogene Erwartungen erfüllende Stilistik. ${ }^{9}$ Auch ein Problembewusstsein für den medialen

\footnotetext{
9 Diese Problematik verdeutlicht sich gegenwärtig insbesondere in der vor allem bezogen auf Bühnenliteratur zunehmend nennenswerten niederdeutschen Übersetzungsliteratur. Die Übersetzer können vielfach nur mit gröBeren Kompromissen Nuancen der Ausgangssprache in die niederdeutsche Fassung übernehmen und erzielen auch beim Publikum nur bedingt den gewünschten Effekt. Für den Hinweis auf das für die vorliegenden Frage-
} 
Übergang von der Mündlichkeit zur Schriftlichkeit haben nur wenige Autoren ausgeprägt, da vielfach kolportiert wurde, man würde Mündlichkeit aufgreifen - tatsächlich wird jedoch grundsätzlich die Existenzform Standardschriftsprachlichkeit imitiert, und sei es nur wegen einer Orientierung an orthographischen Prinzipien. Dass dennoch - wie oben angeführt - ein dialektologischer Informationswert von mundartliterarischer Schriftlichkeit ausgeht, liegt vornehmlich an den jeweiligen dialektologischen Fragestellungen, die in der Regel Einzelmerkmale (wie zum Beispiel Lexeme) aus diesen Texten isolieren lassen, aber bisher in der Regel keine vollständigen Strukturen ihrer literarischen Quellen auswerten und bewerten (cf. Elmentaler 2015, aber auch Jørgensen 1934). ${ }^{10}$

Mundartliche Literatursprachen haben somit unterschiedliche Funktionen ausgeprägt. Zu unterscheiden sind eine ästhetische, eine dokumentierende, eine philologische, eine vermittelnde, eine sprachpflegende, eine ideologisierende, eine volkstümliche und eine unterhaltende Funktion. Funktionen eines Textes können wechseln und unter gegenseitigem Einfluss stehen. Sie zeigen sich in der sprachlichen Gestaltung; insbesondere in der Genauigkeit der Dialektabbildung. Diese Aspekte werden an Texten greifbar, die eine Funktion besonders deutlich ausprägen. So legte die Lyrikerin Waltrud Bruhn (1936-1999) niederdeutsche Texte vor, deren sprachlich-ästhetische Funktion alle weiteren Funktionen überlagert, was zu literarisch außergewöhnlich überzeugenden Texten führte, die jedoch kaum rezipiert oder im niederdeutschen literarischen Diskurs angemessen anerkannt wurden (cf. Stiftung F. V. S. zu Hamburg 1989).

Die unterschiedlichen literarischen Zielsetzungen erbrachten auch unterschiedliche sprachliche Ausgestaltungen, die grammatische, lexikalische und orthographische Varianz ausprägten. Dialektliteratur hat ein hohes Variationspotential, da zwar die ungefähren grammatischen Grenzen einer Mundart zu bedenken, aber keine bestehende Normierung und das von ihr geprägte Sprachverständnis auszuhebeln sind. Somit gründen die wechselnden Profile dialektaler Literalität auf wandelnden Zielsetzungen und Zielgruppen sowie den zunehmend an den sprachlichen Wandel zum Hochdeutschen angepassten Formen dialektaler Schriftlichkeit. Experimentellere Umsetzungen lassen sich temporär in der zweiten Hälfte des 20. Jahrhunderts ebenfalls finden, doch blieb es bei Einzelbeispielen (cf. Anm. 5).

\subsection{Zur Lage niederdeutscher Literatur im 19. Jahrhundert}

Die Rezeption von niederdeutscher Literatur im 19. Jahrhundert unterscheidet sich von ihrer Wahrnehmung im 21. Jahrhundert in einer sehr grundsätzlichen Art und Weise. Mundartliteratur, die auf eine nach heutigem Verständnis nahezu intakte und kompetente Sprechergemeinschaft traf, wirkte anders als Mundartliteratur, die als exotisches Vehikel einer regionalen Selbstvergewisserung innerhalb eines in Richtung Standardsprachlichkeit verschobenen Varietätenspektrums existiert: Den Umbau regionaler Varietätenspektren konnte Mundartlite-

stellungen fruchtbare Problemfeld von Übersetzungsliteratur und der dabei als virulent hervortretenden mangelnden stilistischen Varianz des Niederdeutschen sei Willy Diercks herzlich gedankt (cf. zur niederdeutschen Stilistik Meyer 1923/1983: 71-74 und öfter, sowie Thies 2011).

10 Eine umfangreiche Studie zur sprachlichen, variantenbezogenen Analyse literarischen Textmaterials hat Viola Wilcken (2015) mit ihrer Arbeit über die hochdeutsch basierte, historische niederdeutsch-hochdeutsche Sprachkontaktvarietät Missingsch vorgelegt, deren für lange Zeit breite Verwendung als Literatur- oder auch Komödiensprache ein aufschlussreiches Korpus für die Sprachuntersuchung bieten konnte.

ISSN 1615-3014 
ratur zu keinem Zeitpunkt beeinflussen. Auch die didaktisch orientierte Vermittlungsfunktion neuniederdeutscher Schriftlichkeit ist entwicklungsphasenbedingt. So gab es frühe Autoren, insbesondere um 1900 nach der ersten Phase der Etablierung, die ihre Texte lediglich den Sprechern der von ihnen verwendeten niederdeutschen Varietät vorbehalten wollten (cf. Langhanke 2012a, 2015a zu dem westfälisch-lippischen Mundartdichter Korl Biegemann). Diese ausschließende Definition von niederdeutscher Schriftlichkeit steht in klarer Opposition zur integrierenden Funktion von Standardschriftsprache. Gegenwärtig verfolgen viele Texte den Anspruch, Rezipienten über gut erschließbare Formen und unterhaltsame Inhalte zur niederdeutschen Sprache hinzuführen. Auch die Pioniere neuniederdeutscher Literatur sahen eine Vermittlungsfunktion, allerdings mit anderen Voraussetzungen, denn ihr Hinweis auf Sprachgefährdung war noch nicht begleitet vom gegenwärtig erfahrbaren Verschwinden der Dialekte in der Mündlichkeit. Sie konnten von besseren Sprachkenntnissen der Leser ausgehen, denen weniger ein grundsätzliches Sprachverständnis, sondern vielmehr eine neue, prestigegeleitete Spracheinstellung zu vermitteln war. Jenseits der zeitbezogenen Einschätzungen gilt, dass Schriftlichkeit immer eine didaktische Funktion übernimmt und dialektaler Literalität ein Vermittlungsgedanke inhärent ist; dieser wird im 5. Abschnitt dieses Beitrags vertieft.

Den Entwicklungen im 19. Jahrhundert ist in diesem Kontext ein deutliches Standardisierungsstreben zuzuordnen (cf. auch Langhanke 2017/im Druck), das für die führenden niederdeutschen Autoren zeitweise bestimmend war. Es wird im Folgenden als spezifische Problematik des Transfers von der Mündlichkeit zur Schriftlichkeit vor der Folie einer ausgebauten Standardsprache als historischer Diskurs näher beleuchtet, um die Zielsetzungen, die Möglichkeiten und die zeitgenössischen, bis heute nachwirkenden Ergebnisse einer dialektologisch, sprach- und literarhistorisch sowie didaktisch bedeutsamen Schriftsprachentwicklung voneinander abzugrenzen.

\section{Der niederdeutsche Standardisierungsdiskurs im 19. Jahrhundert}

Der Diskurs über die Reliterarisierung des Niederdeutschen im 19. Jahrhundert betrifft vornehmlich die Ausprägungen und Funktionen seiner Literatursprache (cf. Möhn 1972; Stellmacher 1978; Langhanke 2015a), die unter Umständen auch für weitere schriftsprachliche Anwendungskontexte nutzbar gemacht werden können (cf. Stellmacher 1996: 502-504; Lindow et al. 1998: 311-357; Rüschenschmidt 2006). In der ersten Hälfte des 19. Jahrhunderts entstanden zahlreiche neue Schriftsprachen in Europa (cf. Fredsted 2015: 9). Es ist die Zeit der beginnenden Nationalstaaten, in der eine verbindende Sprachlichkeit zu einem entscheidenden Faktor im zugehörigen Diskurs wurde (cf. Kloss 1987: 104-105). „Die romantischen und nationalistischen Strömungen gaben den Anstoß“ für die Entwicklung von schriftsprachlichen Qualitäten auch kleiner und regionaler Sprachen (Fredsted 2015: 9). Zwischen der nachträglichen Aufwertung mittelniederdeutscher Schriftlichkeit und den Prozessen einer Reliterarisierung des Niederdeutschen im 19. Jahrhundert besteht ein unmittelbarer Zusammenhang, der die neuniederdeutschen „Literaturmundarten“ aus den Schriftformen weiterer Mundartliteraturen zumindest konzeptionell klar hervorragen lässt. Der Ausgangspunkt der erneuten Verschriftlichung liegt in der Entwicklungsgeschichte der hochdeutschen Standardschriftsprache begründet. Deren Entwicklung gilt um 1800 unter den Gesichtspunkten sprachlicher Reichweite, sprachlich-grammatischen Ausbaus und sprachlichen Kulturwertes als abgeschlossen (cf. von Polenz 2013: 2), so dass sich Raum bot, den vernachlässigten regi-

ISSN 1615-3014 
onalen Sprachformen neue Aufmerksamkeit zukommen zu lassen. Fast noch deutlicher als bei der Herausarbeitung der schriftlichen Hochsprache ist der Einfluss von Einzelpersönlichkeiten zu verzeichnen. Für das Niederdeutsche übernahm insbesondere Klaus Groth (1819-1899) die Rolle des reflektierenden Dichters, der sich seiner Tragweite und sprachlichen Sendung bewusst ist und sein Schreiben nicht als „naiv“, sondern als vollkommen programmatisch versteht (cf. zu Groth/Bichel/Hartig 1994; Langhanke 2015b). Ab 1852 erzielte er mit seiner Lyriksammlung „Quickborn“ (cf. Groth 1852/1853) einen dauerhaften Erfolg und trat bald mit theoretisch-reflektierenden Texten zur neuniederdeutschen Schriftlichkeit hervor, die im Folgenden - thematisch und in der Regel nicht chronologisch sortiert - zur Sprache kommen werden:

Angefüllt so von allerlei Wissen und Können, kam mir in den vierziger Jahren zum Bewußtsein, welch ein poetischer Schatz im Plattdeutschen stecke, ungehoben bis dahin, ja bedroht zum Unheil für die Heimat und die große deutsche Literatur durch Unwissenheit und verflachenden Liberalismus. [...] Gesucht werden mußten all die verschiedenen Töne, die ich, der erste, in plattdeutscher Sprache angeschlagen habe; ob dichterische Kraft vorhanden war, frisch und frei in ihnen zu singen, das ist eine vom Wissen und Studium ganz unabhängige Sache.

(Groth 1889/1981: 41)

Vordringliches Ziel von Groths sprach- und literaturtheoretischen sowie autobiographischen Schriften, die das Korpus der Auswertung bilden (cf. die Primärquellenauflistung im Literaturverzeichnis als vollständigen Überblick), ist eine gleichberechtigte Positionierung des Niederdeutschen neben dem Hochdeutschen, ohne dessen erreichte Position zu schwächen. Dieser Spagat gelingt durch spezifische Domänenzuweisungen und wiederholt erneuerte Betonungen der Eigenständigkeit des Niederdeutschen: „Das Plattdeutsche fühlte sich nicht als Dialekt der Schriftsprache, dazu war sie ihr im Bau und Klange zu fremd, es fühlte sich mehr als wirkliche Sprache ihr gegenüber, mit einer Reihe Mundarten unter sich." (Groth 1872/1981, Der Dichter und das Platt: 203) Mit Recht erkennt Groth einen früheren Verschriftlichungsversuch von Johann Heinrich Voß (1751-1826) als niederdeutschspezifisch, da dessen Versuch, an ältere Formen anzuknüpfen und darüber eine neue Ausgleichsform zu schaffen (cf. Bichel 1973/1974, Langhanke 2015a), nur vor dem Hintergrund der gegenwärtig fehlenden, aber historisch angenommenen eigenen niederdeutschen Dachsprache zu verstehen ist. Seine Kritik an Voß richtet sich jedoch eher gegen dessen grammatische „Spracharbeit", ${ }^{11}$ die Groth als einen zu weitgehenden Eingriff des einzelnen Dichters in die grammatisch und lexikalisch gegebene niederdeutsche Sprachlichkeit empfindet, anscheinend gelenkt von einer altphilologischen Tendenz, die auf den Gelehrten und Literaten Voß zutrifft:

Voß nahm dazu nicht seinen heimischen Dialekt, den Mecklenburger oder den hannoverschen der Elbmarsch, den er vom langen Aufenthalt dort kennen mußte, oder den holsteinischen, nein, die Volksmundart hatte für den Gelehrten weder Dignität noch Reinigkeit, die bekam sie erst durch einige Grammatik und Deklination.

(Groth 1872/1981, I. H. Voß und seine plattdeutsche Buchsprache: 196)

Am Beispiel des Dichterkollegen geht Groth auf die Schwierigkeiten bei der Entwicklung einer schriftsprachlichen Varietät und ihrer grammatischen Markierungen ein. Das Dilemma der adäquaten Erfassung des Niederdeutschen vor dem Hintergrund der weitreichenden groth-

11 Cf. zu diesem Terminus und seiner Bedeutung für die Sprachgeschichte des Deutschen die Studie Hundt 2000.

ISSN 1615-3014 
schen Zielsetzungen für eine neuniederdeutsche Literatursprache offenbart sich im Folgezitat, das Potential und Defizit des Niederdeutschen illustriert:

Das Plattdeutsche hat eine ganz andre Stellung, eine so besondere, daß nichts ähnliches in der Welt ihm an die Seite gestellt werden kann. [...] Doch außerdem steht es nicht bloß als der altertümliche [Dialekt] dem Hochdeutschen gegenüber, sondern es ist eine vollständig ausgebildete, wirkliche Schwestersprache, dem Schriftdeutsch zur Seite, und doch wieder all seinen Eigentümlichkeiten nach zugleich nur Mundart, d. h. recht eigentlich nur gesprochene Sprache.

(Groth 1865/1981, Kronzeugen und andre: 169)

Entscheidend ist die Würdigung als ,eine vollständig ausgebildete, wirkliche Schwesternsprache“, die bei aller damaligen gesellschaftlichen Reduzierung des Niederdeutschen keinen Zweifel an seinen sprachstrukturellen Möglichkeiten und sprachhistorischen Verdiensten aufkommen lassen möchte. Diese Ausgangsposition kann eine Schriftlichkeit und Standardisierungen ermöglichen. Als großer Vorzug des Niederdeutschen gegenüber dem Hochdeutschen wird wiederholt seine sprachliche Natürlichkeit und belebende Ungebundenheit angeführt:

Diese Vorzüge verdankt die plattdeutsche Sprache, wie wir gesehen, zum Teil dem Umstande, daß sie nicht durch Bücher gefesselt, ihr Leben in Wald und Feld, auf Strand und Meer als eigentlich gesprochene Sprache fortführt. [...] Es muss Ihnen jetzt zur Überzeugung geworden sein, daß wir nie wünschen können, daß das Plattdeutsche Buchsprache werde. Aber freilich nutzen wird die Schrift für unsern Zweck als Vervielfältigung unserer mündlichen Rede. Wir bringen damit die Mundart auch über ihre räumlichen Grenzen hinaus [...]. Ich wünschte gerade, dass der Hochdeutsche Plattdeutsch lesen möchte.

(Groth 1858/1981, Zweiundzwanzigster Brief: 124-125)

Auch hier drückt sich eine ambivalente Haltung aus - zwar wird die niederdeutsche Gesprochensprachlichkeit bevorzugt, aber dennoch auch Schriftlichkeit für eine weitergehende, jedoch nicht in Konkurrenz tretende Verbreitung gewünscht. Schriftlichkeit macht das Niederdeutsche über die Grenzen der mündlichen Kommunikation hinaus erfahrbar und wieder abrufbar - auch dafür ist eine Standardisierung notwendig. Bestimmen des Arguments der „neuplattdeutschen Literaturbewegung“, so die Eigenbezeichnung der Gruppe durch einige beteiligte Autoren und zeitgenössische Literaturkritiker (cf. auch Eschenhagen 1860), ist die Wiederbelebung einer historisch bereits existent gewesenen Schriftkultur, deren Schriftlichkeit angeblich eine überdialektale Form des Niederdeutschen konservieren konnte. Diese wird stets bemüht, wenn die sprachstrukturellen Gemeinsamkeiten der niederdeutschen Dialekte betont werden sollen. Im Folgenden wird dieses Problem am Beispiel der Diskussion über einen Gedichtband der mecklenburgischen Autorin Alwine Wuthenow (1820-1908) entwickelt, den der mecklenburgische Autor Fritz Reuter (1810-1874) (cf. zu Reuter Batt 1967a) herausgab und den Groth in der Folge sogleich rezipierte:

Lacht nicht wieder der Holsteiner über den abscheulichen Mecklenburger Dialekt? [...] Während umgekehrt, wenn ich aus dem Buch der Frau A[lwine] W[uthenow] in reinem Plattdeutsch vorlas, es uns allen hier wiederum auffiel, wie genau unsere Sprache nach Kern und Wesen über ihr ganzes Gebiet dieselbe bleibt. So vorgelesen, würden diese Lieder jedem holsteinischen Landmann vollständig vertraut, wie von seiner Mutter gesprochen klingen [...]. Schritt und Gang dieser Sprache, der ganze Bau, die Physiognomie sind unser; das Knochengerüst, die Wortstämme sind gänzlich dasselbe, die Verschiedenheit der Mundarten besteht bloß in einigen durchgehenden Vokal- und Konsonantenveränderungen, die bei einer gereinigten Aussprache im gebildeten Munde auch noch fast völlig wegfallen. [...] Die Stämme sind viele Generationen schon getrennt, die öffentliche Stimme einer gemeinsamen Literatur ist seit Jahrhunderten ver- 
stummt; wenn nun der Mecklenburger zum Braunschweiger, zum Westfalen kommt mit den Klängen seiner Heimat und verstanden wird, ist es nicht, als wenn der Bruder heimkehrt [...]?

(Groth 1858/1981, Sechsundzwanzigster Brief: 136-137)

Groth plädiert hier - unter klarer Abschwächung mundartlicher Unterschiede - für die Möglichkeit einer vereinheitlichten Sprachform, die mit regionalen Färbungen versehen werden könnte. Er argumentiert, dass die verbindenden Elemente, die Teil einer möglichen Standardausprägung des Niederdeutschen sein könnten, gegenüber den regional trennenden Merkmalen überwiegen. Eine neu gestaltete Verbindung wäre die gefundene (cf. zu diesem Terminus Stellmacher 1978: 88) gemeinsame Literatursprache der verknüpften niederdeutschen Mundarten. Neben diese kulturell orientierten Bemühungen müsste für einen tragenden Erfolg einer Sprachförderung politischer Einfluss treten. - Den sehr weitreichenden Ideen flämischer Kulturpolitiker und Autoren (cf. Debus 1996) steht Groth jedoch skeptisch gegenüber, da sie eines zeitpolitischen Rückhaltes entbehren - hier bedenkt er realistisch die Möglichkeiten:

Vielleicht ist die Hoffnung zu phantastisch. So jedenfalls, wenn ein holländischer Gelehrter aus den friesischen Provinzen am Zuidersee mir dieser Tage schreibt: ,Die Gleichheit einer Sprache, die sich über ein so großes Gebiet erstreckt, von Emden bis Königsberg, ist wahrlich äußerst merkwürdig. Dies ist für mich auch ein neuer Beweis für die Geschicktheit, welche die niedersächische Sprache besitzt, um als Schriftsprache eingeführt zu werden, worin sie ihre hochdeutsche Schwester übertrifft. In den Ländern des Norddeutschen Bundes müßte das Niedersächsi-

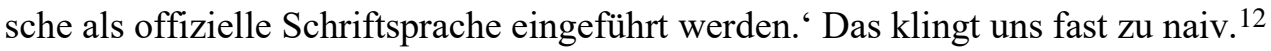

(Groth 1872/1981, Die Politik und die Mundarten: 208)

Das Nachdenken über Schriftlichkeit und Einheitssprache vollzieht sich bei Groth stets mit einem historischen Bewusstsein, das auch die verpassten Möglichkeiten des Niederdeutschen würdigt: „Im Gegenteil sind vielmehr außerdem noch Gründe vorhanden, aus denen man schließen sollte, daß die Entwicklung des Plattdeutschen [statt des Hochdeutschen; R.L.] zur neuen Schriftsprache nähergelegen“" (Groth 1872/1981, Die Mundart und die Pädagogik: 213). Auch diese Beobachtung lässt in der zeitgenössischen Argumentation nur den Schluss $\mathrm{zu}$, dass spätestens jetzt wieder eine niederdeutsche neben die hochdeutsche Schriftsprache treten solle. Allzu weitreichenden Vorstellungen erteilt Groth, der die positive Stellung zur Hochsprache nicht aufgeben möchte, jedoch klare Absagen - so auch in der Auseinandersetzung mit den seltenen Positionen einiger älterer niederdeutscher Autoren, Gelehrter und Sprachverteidiger des späten 16., 17. und 18. Jahrhunderts:

Wenn sie etwas wollten, so wollten sie die Stellung der herandrängenden Schriftsprache für das Plattdeutsche. [...] Und dies unterscheidet sie gänzlich von uns Neuplattdeutschen; wir wollen nicht eins verdrängen, sondern beides erhalten. Wir wollen nicht einmal eine zweite Schriftsprache neben dem Hochdeutsch, sondern wir wollen die Mundart im Platt, wenn nötig auch schriftlich und im Druck, am liebsten gelesen und gesprochen. [...] Wenn Reuter und ich nebst unseren Kollegen ganze Bibliotheken zusammenschrieben, so täten wir der hochdeutschen Literatur keinen Abbruch. Denn wir wollen etwas schaffen, was sich in der Schriftsprache nicht schaffen lässt.

(Groth 1872/1981, Die Mundart und die Pädagogik: 218-219)

\footnotetext{
12 Als Randbemerkung sei notiert, dass der Norddeutsche Bund von 1867 im Jahre 1872 bereits dem 1871 proklamierten Deutschen Reich (dem sogenannten zweiten deutschen Kaiserreich) gewichen war, so dass spätestens nun entsprechende Bestrebungen den Charakter einer vollständigen Utopie haben mussten.
}

ISSN 1615-3014 
Schriftlichkeit erscheint hier einmal als notwendiges Vehikel zum Spracherhalt, dann aber auch als ästhetisch erwünschte Ergänzung zur hochdeutschen Schriftsprache ohne Gefährdungspotential.

Für eine erfolgreiche Standardisierung des Niederdeutschen fehlten somit konkrete sprachliche Aufgaben, für die ein solcher Vorgang im 19. Jahrhundert notwendig gewesen wäre. Das ergänzende und kreative Potential wurde nach 1850 ohne Zweifel geweckt. An die Stelle der ,allgemeinen Schriftsprache“ ist eine ,allgemeine Schreibelust“ getreten, angeregt durch das Interesse an sprachlicher Vielfalt in der eigenen Region:

Wir haben seitdem, wenn auch keine allgemeine Schriftsprache, so doch neben dem neu erwachten Interesse für alle Stammsprachen eine allgemeine Schreibelust an fast allen Ecken und Enden Niedersachsens von Danzig bis Dünkirchen, von der russischen bis zur französischen Grenze, im geraden Gegensatz zur früheren literarischen Schweigsamkeit. Wir zählen schon plattdeutsche Bücher und Schriften nach Dutzenden und manchen guten Namen darunter. [...] Jedenfalls ist es erneutes Interesse.

(Groth 1872/1981, Ende der Mundarten: 236)

Die „ausgebildete Stammsprache“ Niederdeutsch ist in der zeitgenössischen Wahrnehmung Groths zunehmend kulturell abhängig von der neuen Schriftlichkeit. Wenn Schriftsteller den Fortbestand einer Sprache mitgestalten, ist ein standardisierender Einfluss jedoch kaum vermeidbar; zumal dann nicht, wenn Fragen von Spracherhalt und Sprachvermittlung ebenfalls hineinspielen:

Zugleich ist es aber auch [...] die Pflicht jedes Mannes von Einsicht, diesem Vorgange nicht gleichgültig zuzusehen, sondern sein Teil zu tun zur Erhaltung dieser berechtigtsten aller Eigentümlichkeiten, einer ausgebildeten Stammsprache [...] Um wieviel wäre vielleicht schon das Gebiet des Plattdeutschen seit 1850 zusammengeschmolzen ohne das erneute Interesse für die Sprache durch die plattdeutschen Schriftsteller?

(Groth 1872/1981, Männlicher und weiblicher Stamm der Mundarten: 243)

Während Groth eine von dem Germanisten Karl Müllenhoff (1818-1884) und ihm selbst entwickelte niederdeutsche Orthographie zwar zeitlebens als außerordentlich hochstehend einschätzte, wandelte sich jedoch mit den Jahren seine Einstellung zu einer allgemeinen niederdeutschen Schriftsprache. So äußerte er 1885 in der Rezension einer Bibelübertragung: „Der Herausgeber hat mit Recht als Norm seine eigene Mundart gewählt, denn eine Art allgemeingültiges Plattdeutsch, wie J. H. Voß es in seinen Idyllen herzustellen versuchte, führt nur auf Irrwege." (Groth 1885/1981: 321) Allerdings bleibt dieser ungewohnte Ausspruch nicht allein stehen und wird durch die autoritätengestützte Feststellung begleitet, dass

[d]er berühmte Sprachkundige Prinz Lucien Bonaparte [...] den Ausspruch getan [hat], von allen plattdeutschen Mundarten sei die holsteinische (will sagen dithmarsische) die vollkommenste. Dies bezieht sich ohne Zweifel auf den reinen Vokalismus derselben, ein Vorzug, den schon vor langem Professor Müllenhoff gerühmt hat. ${ }^{13}$

(Groth 1885/1981: 321)

So plädiert Groth zwar zum einen für eine sprachlich freie Gestaltung, definiert aber zum anderen seine eigene Mundart als besonders willkommen und schlägt deren literatursprachliche Verwendung in seiner Rezension der Bibel-Übertragung vor: „Die leicht bewerkstelligte Änderung in diesem Sinn [...] würde nach meiner Ansicht eine wesentliche Verbesserung sein.“ 
(Groth 1885/1981: 321) Groth sieht seine eigene Literatursprache als „Leitvarietät“ (Reichmann 1990) an. Dass diese Vorgabe verhältnismäßig wenig Erfolg haben sollte, lag allein in der Konkurrenz durch andere Autoren begründet, denen die grothschen Regeln nicht geschlossen zusagten. - Insbesondere Fritz Reuter, der ab 1853 in mecklenburgischer Mundart publizierte und mit niederdeutscher Erzählprosa nach 1859 große Erfolge erzielte (Ut mine Stromtid, cf. Reuter 1862-1864), behielt die sprachfördernden Zielsetzungen der „neuplattdeutschen Bewegung“" ebenfalls im Blick und zog eigene Schlüsse.

Beherzigen Sie meinen Vorschlag, der - auf mein Gewissen gesprochen! - durchaus nicht die Absicht hat, den einen Dialekt zu zwingen, sich dem andern zu Gunsten aufzugeben; nein! alle sollen sich aufgeben, und zwar zu Gunsten der plattdeutschen Sprache. [...] Durch die von Groth und Müllenhoff im Quickborn nachträglich gemachten Regeln ist viel Unheil entstanden, und wenn jeder plattdeutsche Schriftsteller aus seinem Dialekte sich solche Regeln bilden wollte, dann adieu!

(Reuter an Johann Meyer, Neubrandenburg, 22. 9. 1859, in: Hückstädt 2009a, 420f., hier 420f.)

Reuters inhaltlich dazugehöriger Ausruf ,denn ich liebe meine Sprache mehr als meinen Dialekt" (Reuter 1859/1966-1967: 14) spitzt diesen Gedanken zu, indem er über die Summe der niederdeutschen Dialekte eine niederdeutsche Sprache setzt, die es durch die Rückführung auch auf die historischen Schreibsprachen herauszuarbeiten gelte, so Reuters Idee. Genauere Vorstellungen und literatursprachliche Umsetzungen ergeben sich jedoch auch bei Reuter selbst, der stets seine mecklenburgische „Literaturmundart“" verwendete, neben einigen orthographischen Vereinheitlichungen nicht. Die Setzung einer bestimmten niederdeutschen „Literaturmundart“ als „Leitvarietät" lehnt er, anders als Groth, zudem ohnehin ab:

Es ist daher auch ein vergebliches Mühen, wenn ein plattdeutscher Schriftsteller sein Idiom als das vorzüglichere den übrigen Volksstämmen oktroyieren will, wenn er sich an seinen Schreibtisch setzt und aus seinen vielleicht höchst einseitigen Werken allgemeine Regeln für die Sprache aufstellt [...]. Eine solche, auf der Studierstube gemachte Sprache hat kein Fleisch und Blut, sie hat kein Leben; und könnte ihr dies künstlich eingeblasen werden und gäben die übrigen Idiome ihre Rechte einseitig auf, so wär's das größte Unglück für die Sprache, es wäre ein selbstmörderischer Akt zugunsten einer höchst zweifelhaften Autorität.

(Reuter 1859/1966-1967: 11)

Es gehört zu den Besonderheiten der niederdeutschen Literaturgeschichte, dass ausgerechnet Reuters „Literaturmundart“ wegen der großen Erfolge seiner Texte jedoch temporär im 19. Jahrhundert ab ca. 1860 und auch noch im frühen 20. Jahrhundert die Funktion einer solchen „Leitvarietät“ fraglos zukommen sollte, ohne dass ihr Urheber das jemals forciert hätte. Groths „Literaturmundart“" ist hingegen trotz expliziter entsprechender Bemühungen des Dichters nur vereinzelt aufgegriffen worden, auch seine Orthographie konnte sich nicht mit sonderlichem Erfolg durchsetzen (cf. zu diesen Bemühungen das programmatische Vorwort Groth 1878 und die Broschüre [Groth] 1876). Dialektale Vielfalt in der Literaturproduktion wurde sowohl von Groth als auch von Reuter anfänglich - zumindest in den 1850er Jahren als Problem erachtet (cf. Reuter an J. H. Mertens, Neubrandenburg, 16. 12. 1859, in: Hückstädt 2009a: 431f., dort 432). Bei Reuter stellte sich jedoch bereits zu Beginn der 1860er Jahre ein Einstellungswandel ein, der unter anderem mit einem Argument Jakob Grimms begründet wird, das dieser Reuter brieflich mitteilte - dieser Brief ist zudem auch ein Ausweis der überaus weitreichenden Netzwerkbildungen von Groth und Reuter. Grimm argumentierte als 
Sprachhistoriker und Dialektologe und nicht als Befürworter einer neuen allgemeinen niederdeutschen Schriftsprache - der er auch nicht war:

Auch den Nörgeleien über falsche und inconsequente Orthographie weiß ich nichts anders zu erwidern, es sei denn, daß ich mich auf die Autorität eines Mannes berufe, der obgleich todt, doch ewig leben wird, auf Jakob Grimm, der mir den Rath gab, meine Orthographie nicht zu ändern, ,denn', sagte er, ,dadurch, daß Sie so schreiben, wie zu Ihrer Zeit in Ihrer Gegend gesprochen worden ist, geben Sie einem späteren Forscher ein deutlicheres Bild, als wenn Sie sich einer thörichten, projectirten Verschmelzung aller niederdeutschen Dialekte anbequemen wollten'. ${ }^{14}$

(Reuter an einen Unbekannten, Eisenach, 15. 2. 1864, in: Hückstädt 2009b: 189f., hier 189f.)

Diese „thörichte, projectirte Verschmelzung aller niederdeutschen Dialekte“ (die hier verkürzt wird auf orthographische Fragen) ist tatsächlich nie zur Ausführung gelangt - so wie auch ein für das Jahr 1861 geplanter Dichterkongress zum Thema nicht stattfand (cf. Langhanke 2015b: 343) - und blieb in ihrer konkreten Durchführung stets undeutlich. Reuter gelangte in seinen wenigen Modellierungen des Themas nicht über Schlagworte hinaus und stand mit ihnen zunächst in einem Gegensatz zu den dialektologischen Interessen seiner Zeit (cf. Abschnitt 2 sowie Langhanke 2011a). - Tatsächlich aber sind sowohl Groth als auch Reuter über ihre Texte zu wichtigen Gewährsleuten ihrer regionalen dithmarsischen beziehungsweise mecklenburgisch-vorpommerschen Mundarten geworden, wie zahlreiche dialektologische Publikationen belegen (cf. stellvertretend Jørgensen 1934 und zur Methodik Elmentaler 2015 und Rohdenburg 1991, 2002). Die neuniederdeutsche Schriftlichkeit entwickelte sich zu einer vielfältigen Gruppe von „Literaturmundarten“ einzelner Mundartregionen. Um 1860 jedoch hatte der Vereinheitlichungs-Gedanke Reuters und auch Groths eine gewisse Attraktivität erreicht und wurde von literaturkritischer und philologischer Seite unverändert aufgegriffen, wie ein Zitat des bereits erwähnten zeitgenössischen Publizisten Eschenhagen (ebenfalls diskutiert bei Langhanke 2015a, 2016a) überzeugend aufzeigen kann:

[S]o dürfte es doch wünschenswerth sein, dass durch Verschmelzung und gegenseitige Ergänzung der Dialecte eine allgemeine plattdeutsche Schriftsprach zu Wege gebracht würde, die nicht die hochdeutsche Schwester verdränge, und über diese gestellt würde, aber für diejenigen Stoffe, für welche sie mehr als das Hochdeutsche geeignet, und auf welche sie also ein Recht hat, ein vollkommeneres Gewand abgäbe, was keiner der Dialecte allein vermag.

(Eschenhagen 1860: 21)

Das „vollkommenere Gewand“, „was keiner der Dialecte allein vermag“, bringt eine nennenswerte Ergänzung zu den Ausführungen Reuters, indem nahegelegt wird, dass für die Übernahme spezifischer schriftsprachlicher Funktionen durch das Niederdeutsche zunächst „Spracharbeit“ notwendig sei, die eine standardisierte Sprachform schaffe, die es auch in dieser Hinsicht mit der „hochdeutschen Schwester“ aufnehmen könne. Diese Ansicht haben die niederdeutschen Autoren nicht geteilt und der hochdeutschen Standardschriftsprache ihre individuellen niederdeutschen „Literaturmundarten“ erfolgreich an die Seite gestellt. Folglich hat die wirkungsvolle Reliterarisierung des Niederdeutschen zwar eine neue Wahrnehmung der Sprache und eine neue Literaturtradition begründet, aber eine neuniederdeutsche Standardschriftsprache konnte, abgesehen von der langjährig gültigen Vorbildfunktionen der reuterschen „Literaturmundart“, bisher nicht ausgeprägt werden. Unter den niederdeutschen Au-

14 Das Original des zitierten Briefes von Grimm an Reuter ist nicht auf uns gekommen oder hat sich gegenwärtig noch nicht wieder angefunden. 
toren war und ist eine neuniederdeutsche Standardschriftsprache in aller Regel auch nicht erwünscht, dennoch bestimmte sie als Idee zunächst die sprachliche und literarische Diskussion mit, da ein starkes Motiv und Argument der vorgestellten Konzeption der Rückbezug auf die mittelniederdeutschen Schreibsprachen war, die zeitgenössisch vielfach als einheitliche Schriftsprache aufgefasst und gewertet wurden (cf. den inzwischen veränderten Erkenntnisstand bei Peters 1995/2012; Peters 2017/im Druck; sowie bündelnd auf Peters Bezug nehmend Langhanke 2017/im Druck). Fritz Reuter sah lediglich temporär konkrete Umsetzungsmöglichkeiten:

Mein Vorschlag geht in nuce dahin: auf die alte Sprache zu rekurriren und dieser zu Gunsten alle Unarten und Unwesentlichkeiten der Dialekte aufzugeben, dies aber nicht mit einem Schlage zu thun, um unserm Lesekreis nicht plötzlich fremd zu werden, sondern allmählich [...].

(Reuter an J. H. Mertens, Neubrandenburg, 16. 12. 1859, in: Hückstädt 2009a: 431f., hier 432)

Reuters didaktischer Ansatz, der sich auch in orthographischen Zugeständnissen an das Hochdeutsche zeigt, beweist seinen damaligen Anspruch, tatsächlich eine einheitliche niederdeutsche Schriftsprache erfolgreich zu installieren. Seine Vorstellungen von einer unterschiedliche Sprachformen vereinenden oder verschmelzenden Schriftsprache, die Groth zugunsten einer gegebenen „Leitform“ („Leitvarietät“) mehrfach verneinte, spiegelt zwar nicht die historische sprachliche Realität des 13. bis 16. Jahrhunderts, aber die sprachliche Wunschvorstellung des 19. und 20. Jahrhunderts wider (cf. zu diesem historischen sprachhistorischen Konzept einschlägig Peters 1995/2012: 430):

Da, wo die plattdeutsche Sprache aufhörte, Schriftsprache zu sein, ist das Band zuerst gerissen, da muss vernünftigerweise wieder angeknüpft werden; da könnte man den Knoten schlagen, der alle Dialekte wieder zu einem Ganzen verbände. [...] Wir müssen daher nicht von dem Riss ausgehen, sondern von unseren heimatlichen Ufern aus dahin vorgehen [...], wir müssen das Unwesentliche über Bord werfen und das Zufällige der Aussprache dem Leser überlassen.

(Reuter 1859/1966-1967: 12f.)

Der Handlungsentwurf sieht die Möglichkeit der Rückführung einer gegenwärtigen, an Einzelmundarten orientierten Schriftlichkeit auf die historische niederdeutsche Schriftlichkeit vor, die hier jedoch als überregional behauptet und betrachtet wird. So erklärt sich auch das Bild des sprachlichen Risses, das Reuter wiederholt bemüht, um eine wiederherstell- und erneuerbare Konstanz niederdeutscher Schriftlichkeit zu betonen:

Jeder plattdeutsche Schriftsteller muß von seinem heimischen Gestade Abschied nehmen, das große Sprachkabel sorgfältig verfolgen bis an jenen Riß und dort [...] anknüpfen. [...] Da, wo die plattdeutsche Sprache aufhörte, Schriftsprache zu sein, ist das Band zuerst gerissen, da muß vernünftigerweise zuerst wieder angeknüpft werden; da könnte man den Knoten schlagen, der alle Dialekte wieder zu einem Ganzen verbände.

(Reuter 1859/1966-1967: 12)

Bei aller historischen Ungenauigkeit und philologischen Unmöglichkeit dieser Aussage verdeutlicht sie doch, welche Entwicklungsmöglichkeiten um 1860 gesehen und als attraktiv erachtet wurden. Im 19. Jahrhundert wurde die Basis einer langen Wirkungsgeschichte niederdeutscher Literalität bis in das 21. Jahrhundert geschaffen. Dass für eine langfristige Wirksamkeit kulturhistorische Fundamente gesucht wurden, erwies sich als bewährtes Verfahren auch unter rezentem sprachpolitischen Gesichtspunkt ist zu verzeichnen, dass der Verweis auf die historische Wirksamkeit des Mittelniederdeutschen eines der entscheidenden Argumente 
für die Anerkennung des Niederdeutschen als Regionalsprache im Rahmen der Europäischen Charta der Regional- oder Minderheitensprachen war (cf. einschlägig Menkes Terminus der „historischen Einzelsprache“; siehe dazu Menke 1998: 184; 2001). Diese sprachpolitischen Förderkulissen können auch in einen Zusammenhang mit den Perspektiven der skizzierten neuniederdeutschen Schriftlichkeitstradition ab dem 19. Jahrhundert gesetzt werden.

\section{$4 \quad$ Perspektiven niederdeutscher Literalität}

Für die im 19. Jahrhundert etablierte niederdeutsche Literalität sind in der Gegenwart des 21. Jahrhunderts drei Perspektiven zu unterscheiden: (1) Noch gibt es die zumindest grundsätzliche Abbildung kleinräumlicher niederdeutscher Sprachlichkeit über das geschriebene Feld der Mundartliteratur, in welchem auch die Darstellung grammatischer Vielfalt gegenüber den geregelten hochdeutschen standardsprachlichen Strukturen existent bleibt. - (2) Zudem ist eine normierende Funktion der Literalität für institutionalisierte spracherwerbsbezogene Lehr-Lernprozesse auszumachen. Diese ist ausbaufähig. - (3) Schließlich sind die vollkommen unabhängigen kreativen Verwendungsweisen des Niederdeutschen in den neuen sozialen Medien zumindest gegenwärtig als produktiv nachweisbar (cf. Reershemius 2015b); ihr Anteil am schriftsprachlichen Gesamtaufkommen des Niederdeutschen ist jedoch gering.

Wenigstens vier Funktionen erfüllt niederdeutsche Literalität gegenwärtig nicht mehr: (1) Eine detaillierte und flächendeckende Abbildung von kleinräumlicher Sprachlichkeit ist nicht mehr gegeben, da zunehmend weniger niederdeutsche „Literaturmundarten“ aktiv beziehungsweise produktiv gebraucht werden, so dass historische „Literaturmundarten“ von noch aktiven rezenten Gebrauchsformen zu differenzieren sind. - (2) Auch eine Selbstvergewisserung der dialektalen Sprechergemeinschaft über Texte in ihrer Sprachform, also die unwillkürliche, gleichsam „volkstümliche“ Rezeption der Texte entfällt, während sie für die ältere Mundartliteratur neben der bürgerlich-akademischen reflektierten Rezeption konstituierend war (cf. Groth 1864, 79). - (3) Zudem fällt die produktive Erschließung von Sprachregionen in areal-horizontaler Hinsicht fort. Das Zeitfenster für entsprechende Entwicklungen - gemeint ist die schriftsprachliche Ertüchtigung dialektaler Sprachräume - ist in Norddeutschland für den ungefähren Zeitraum 1780 bis 1990 anzusetzen (cf. auch Argumente bei Peters 1998/2012). - (4) Und schließlich ist die Förderung dialektaler Sprechsprachlichkeit eine nur noch bedingt erfüllte Funktion niederdeutscher Schriftlichkeit. Ob ältere Mundartliteratur eine Förderung dialektaler Mündlichkeit erreichte, bleibt ebenfalls ungewiss. Zu unterscheiden ist Sprachförderung, die auf Verbesserung bestehender dialektaler Mündlichkeit oder ihre Verfestigung abzielt, von Förderung, die eine Wiederbelebung von niederdeutscher Mündlichkeit vorsieht. Für beide Ziele kann Mundartliteratur eine stützende Funktion übernehmen, da sie grammatische Formen und Wörter in Anwendung bietet und zudem Prestigesteigerung bewirkt. - In der Zusammenschau erweisen sich somit zahlreiche historische Wirkungspotentiale von niederdeutschen ,Literaturmundarten“ als gleichsam abgehängt.

Die weitreichende Erschließung horizontaler, bedingt auch vertikaler Sprachlagen oder Varietäten des Niederdeutschen durch Mundartliteratur ist ein historisches Phänomen (cf. Punkt 3 im vorherigen Absatz). Autoren in verschiedenen Regionen bildeten Schriftlichkeit aus und sorgten für „literaturmundartliche“ Vielfalt in der Fläche. Produktiv betrachtet ist dieser Umstand historisch, da in vielen Regionen dialektale Literalität wieder zum Erliegen gekommen ist. Unter arealer Perspektive ist eine Konzentration auf ausgewählte „Literaturmundarten“ zu 
beobachten, in denen die Weitergabe sprachlichen und schriftsprachlichen Wissens durch höhere Sprecherzahlen noch ermöglicht ist. - Die erfolgsnotwendige Attraktivität einer „Literaturmundart" besteht aus dem Reüssieren einzelner Texte; so haben Fritz Reuters Romane der mecklenburgischen „Literaturmundart“ zwischen 1860 und 1950 und besonders um 1900 zu großer Popularität verholfen (cf. Batt 1967; Langhanke 2016). Gegenwärtig ist die Etablierung der orthographischen, lexikalischen und grammatischen Normierungsvorschläge des SASS-Wörterbuches (Kahl/Thies 2013) und der SASS-Grammatik (Thies 2011) bestimmend, zumal niederdeutsche Schreibwerkstätten auf dieser Grundlage arbeiten (cf. dazu auch Goltz 2015). Diese von der Fehrs-Gilde als sprachpflegender Vereinigung herausgegebenen Nachschlagewerke repräsentieren das Bemühen von Teilen der kulturell um das Niederdeutsche bemühten Gruppe, zu quasi-offiziellen Regelungen zu gelangen, die eine klare Dichotomie von richtig und falsch auch für denjenigen ermöglichen, die über kein gesichertes Sprachgefühl mehr verfügen.

Das Verhältnis niederdeutscher „Literaturmundarten“ zu Mündlichkeit und Schriftlichkeit hat sich verschoben. So ist ein Wandel von der Imitation lebendiger Mündlichkeit in der Schriftlichkeit hin zu Textvorlagen für gesteuerte Mündlichkeit feststellbar, denen konkret betrachtet eine hilfreiche Funktion in Sprachvermittlungssituationen zukommen kann oder auch zunehmend zukommen wird (cf. zu diesen Entwicklungen Goltz 2014, Bendixen 2016).

Neben der Imitation standardsprachlicher Schriftlichkeit durch dialektale Literalität steht die Orientierung an mundartliterarischer Tradition. Die Entwicklung schriftsprachlicher Traditionen innerhalb von dialektaler Literalität bedeutet fortschreitende Abkehr von der Ausgangsorientierung an dialektaler Mündlichkeit. Dass Traditionsentwicklung mit fortschreitender Normierung und späterer Rückwirkung auf rezente Formen der Mündlichkeit einhergehen kann, ist den Wechselprozessen zwischen dialektaler Mündlichkeit und Schriftlichkeit im Spannungsfeld von Sprachverlust und gesteuertem Spracherhalt eingegeben.

Einen lebendigen Diskurs über niederdeutsche Schriftlichkeit und ihre Optionen bieten Publikationen noch bis in die 1980er Jahre hinein. Das Sprechen über niederdeutsche Schriftlichkeit ist dabei das Nachdenken über niederdeutsche Dichtung. Dichterischer Gestaltungsanspruch ist Notwendigkeit und Existenzgrundlage für niederdeutsche Schriftlichkeit und wird traditionell mit dem Stichwort „,sprachschöpferisch“ in Verbindung gebracht, nüchterner wäre der Terminus „Sprachausbau“. Ein Hauptanspruch niederdeutscher Dichtung, wie ihn unter anderem der Autor Moritz Jahn (1884-1979) in der Mitte des 20. Jahrhunderts vertrat, ${ }^{15}$ ist die Übertragung der schwindenden sprachschöpferischen Energie alltäglicher Sprechsprache auf Schriftsprache. Das bedeutet jedoch auch, sich von einer Abbildung des tatsächlich gesprochenen, auch einem Wandel und hochdeutschem Einfluss unterworfenen Dialekts abzuwenden und den Ausbau einer literarischen Kunstsprache grammatisch und lexikalisch voranzutreiben. Moritz Jahn möchte dafür in den 1960er Jahren noch den „Geist des Plattdeutschen" beschwören, eine bildhafte Erfassung idiomatischer Strukturen des Niederdeutschen: „[...] [E]inen neuen Aussagebereich für eine Sprache zu gewinnen, ist ja schon eine sprach-

\footnotetext{
15 Jahn gehört zu den wenigen niederdeutschen Autoren des 20. Jahrhunderts, die reflektierende Texte über ihre Vorstellung von niederdeutscher Sprache und Literatur publiziert haben und ist daher stärker in den Fokus der Forschung gerückt als andere niederdeutsche Autoren; cf. dazu die einschlägigen Sammelbände Stellmacher 1986 und Rohse/Stellmacher/Hinrichs/Semmelroggen 2011.
}

ISSN 1615-3014 
schöpferische Leistung, die sich natürlich dann auch in der Bildung neuer, aus dem Geist des Plattdeutschen heraus geborener Wörter äußern kann.“" (Jahn 1964: 56-57) Eine weitere Äußerung Jahns schließt an Vorstellungen Klaus Groths an (siehe unten), wenn er vor dem Hintergrund grammatischer und lexikalischer hochdeutscher Einflüsse auf niederdeutsche Sprechsprache die Einschätzung äußert: „Das ist ein trauriger Zustand, und eben darum ist plattdeutsche Dichtung, die wirklich Dichtung ist, nötig, weil sie einer zukünftigen Zeit die Sprachdenkmäler schafft.“ (Jahn 1964: 58) Es ist jedoch deutlich, dass Jahn einer niederdeutschen Mündlichkeit keine Zukunft einräumt. Steuerungen niederdeutscher Schriftlichkeit sind in der Lexik gut greifbar, so dass neue Texte in der Regel gern über ihre Wortneuschöpfungen beurteilt werden. Als besonders kreativ gilt Christian Holsten, der 1964 ausführt: „Ik glööf aber, dat dat in 'n Plattdüütschen noch wat lichter to is as in 'n Hochdüütschen, vonwegen dat dat noch nich so faß liggt as Schriftspraak dör de Jahrhunnerte, sondern dat is noch frisch un neet. Un de Lüer weet ok faken nich, dat dat 'n neet Wort is. De denkt, dat is in de Landschaft begäng, wo de, de dat schreben hett, herstammen deit.“ (Holsten 1964: 48) Somit eröffnet die geringe Reichweite und Bekanntheit einer bestimmten niederdeutschen Mundart (und ihrer zugehörigen „Literaturmundarten“) zahlreiche Möglichkeiten für Neuschöpfungen. Den Ausweg zeitgenössischer niederdeutscher Mündlichkeit, neue Zusammenhänge durch die Übernahme von Ausdrücken aus dem Hochdeutschen zu klären, die sich auf diese Weise von parallel existenter niederdeutscher Mündlichkeit entfernt, will Holsten für die Schriftlichkeit nicht akzeptieren. Schriftlichkeit komme die Aufgabe zu, die Sprache weiter zu entwickeln und der Mündlichkeit hilfreiche Alternativen zu bieten (cf. Holsten 1964: 48f.). Diese spracherhaltende Perspektive war zumindest in ihrem theoretischen zukunftsweisend, doch ihr sprachdidaktischer Erfolg harrt noch einer empirischen Betrachtung - so zum Beispiel durch Vergleiche von vielfältig vorkategorisierten Wortschatzbeständen in den niederdeutschen Texten einer bestimmten Region im Verlaufe der zweiten Hälfte des 20. Jahrhunderts; die weiteren grammatischen Ebenen müssen hinzutreten. ${ }^{16}$

Unterschiedliche Einschätzungen lassen sich zu der Frage finden, ob niederdeutsche Schriftlichkeit jenseits niederdeutscher Mündlichkeit denkbar sei. Selten sind diese Positionen ideologiefrei. Während ältere neuniederdeutsche Schriftlichkeit auf Mündlichkeit basiert, nimmt dieser Bezug im Laufe der Entwicklungsgeschichte ab, so dass gegenwärtig der Vorwurf im Raum steht, es könnte im Rahmen von Vermittlungskontexten oktroyierte niederdeutsche Schriftlichkeit geben, deren produktive und rezeptive Verwendung „top down“ verordnet und nicht „bottom up“ entwickelt sei (cf. Fredsted 2015, 21-22). Normierte und didaktisierte niederdeutsche Schriftlichkeit kann die alte idealistische Forderung nach echter plattdeutscher Dichtung im Sinne der Anwendung individuell-erstsprachlich erlebter Sprechsprache als Literatursprache nicht mehr erfüllen. Merkmalsausgleich tritt vor Merkmalserhalt.

Ein abschließend noch zu ergänzender, bisher in diesem Beitrag wegen seiner in mancher älteren Forschung bisweilen zu einseitig diskutierten Folgen nicht reflektierter wirkmächtiger Disput zwischen Klaus Groth und Fritz Reuter Ende der 1850er Jahre gibt Einblick in traditionelle, jedoch auch fortgesetzt gültige Profile von Mundartliteratur (cf. Langhanke 2015a). Die Autoren vertraten eine unterschiedliche Auffassung von niederdeutscher Schriftlichkeit,

16 Entsprechende korpusgestützte Untersuchungen sind durch den Autor und im Rahmen von Abschlussarbeiten Flensburger Studierender in Vorbereitung.

ISSN 1615-3014 
die durch sozialen Stand und die historische Entwicklung der Sprachregionen bedingt ist (cf. dazu überzeugend Batt 1958, 1967b): Groth wollte die autochthone ländliche Bevölkerung über eine Ästhetisierung („Veredelung“) ihrer ursprünglichen niederdeutschen Mundart zu einer idealisiert-erhöhenden Betrachtung der eigenen kulturellen Werte führen. Diese Erhöhung bedeutete auch Abkehr von sprachlicher und lebensweltlicher Realität. Reuter wollte reales Sprachleben spiegeln und Sozialkritik üben - was eine Illustration schichtenspezifischen Sprechens bedeutete, worauf Groth weitgehend verzichtete. Gleichwohl berühren sich die Konzepte in diesem Punkt - Niederdeutsch ist auch in der literarischen Umsetzung die Sprache der einfachen Bevölkerung, nur erfährt sie bei Groth eine bewusste Poetisierung und erhält bei Reuter einen Anstrich von Realität, wobei er sich der Komik als Vehikel bedient. Während bei Groth im Zuge der Textrezeption weniger gelacht, eher geweint werden darf (wobei diese Pauschalisierung erkennbar verkürzend argumentiert), verwendet Reuter humoristische Brechungen der bisweilen auch tragischen Erzählhandlungen als vermittelndes Element. - Im Vergleich der Sprach- und Inhaltskonzeptionen von Groths und Reuters Texten überwiegen jedoch klar die Gemeinsamkeiten, da der „Parallelismus“ von Lebenswelt und Sprache (Haas 1983: 1639 und öfter) und die schriftsprachliche Orientierung an Strukturen der Standardsprache beide Konzepte eint. Sie beanspruchen bis in die Gegenwart Gültigkeit.

\section{$5 \quad$ Niederdeutsche Schriftlichkeit im Kontext von Sprachvermittlung}

Dialektale Literalität hat sich gelohnt. Ihre Texte sind Beweis einer kulturellen Gestaltungsund Ausdrucksfähigkeit des Dialekts neben der Standardsprache trotz der ideologischen Gefährdung des Bestands einer dialektalen Kultursprache. Dennoch bleibt dialektaler Schriftlichkeit das Unerhörte zu eigen. Ihr bei aller Imitation des Hochsprachlichen eigenständiges Potential gegenüber der verabredeten Einheitlichkeit von Standardschriftsprache als sprachgeschichtlicher Errungenschaft hat stets zu neuen Ausdrucksformen geführt und Autoren für sie interessiert. Diese Wirkung lässt in dem Moment nach, in dem der sprachliche Rückhalt in der Mündlichkeit deutlich schwindet, denn dann ist es nicht mehr möglich und nicht mehr notwendig, für eine Sprechergemeinschaft einzustehen, die sich im mündlichen Sprachalltag gegen standardnahe Sprachformen behaupten möchte und als Trägergruppe einer untergehenden Sprach-, Lebens- und Sachkultur auftritt. Von paralleler dialektaler Mündlichkeit getrennte dialektale Schriftlichkeit kann nur für kurze Frist überleben, die solange dauert, wie der Mundart noch ein memoriales und museales Interesse entgegengebracht wird. Am längsten nachwirken dürfte, wie bei aller je hervorgebrachten Schriftlichkeit, das philologische Interesse - auch weil es nicht zwangsläufig der steten Erneuerung eines Textbestandes bedarf.

Die Feststellung des Unerhörten von dialektaler Literalität steht im Konflikt zur gängigen Wahrnehmung als konservativ-sprachbewahrend. Mit diesem Widerspruch ist ein weiter Teil ihrer bestimmenden Elemente und Spannungsfelder erfasst, obgleich oder gerade weil ihre Existenzformen erstaunliche Heterogenität zeigen. Innovative Lyrik existiert neben sprachpflegerischer Programm- und Tendenzschriftlichkeit sowie zahlreichen auf Situationskomik ausgerichteten Erzähltexten.

Als problematisch erweist sich die Verknüpfung von dialektaler Literalität und sprachlicher Kompetenz. Während der Kompetenzbegriff auf standardsprachliche Schriftlichkeit klar Anwendung findet, bleibt er für dialektale Schriftlichkeit undeutlich. Ob, und wenn ja, welche Kompetenzen wünschenswert sind, ist ebenso undeutlich wie die Steuerung und Weitergabe

ISSN 1615-3014 
dieser Kompetenzen. Der hilfreiche Blick auf Mundartliteraturgeschichte als der einzig fassbaren Tradition von dialektaler Literalität zeigt individuelle Akteure, während sich schriftsprachliche Traditionen kaum ausgeprägt haben. Gegenwärtig ist noch undeutlich, ob dialektale Schriftlichkeit als vermittelte und vermittelnde Kompetenz institutionell gesteuerter Sprachvermittlung gelten wird. Im niederdeutschen Sprachraum sprechen einige bildungspolitische Entwicklungen (stärkere Verankerung des Niederdeutschen an hamburgischen Schulen seit 2010 und an schleswig-holsteinischen Schulen seit 2014; cf. dazu Goltz 2014) für den Ausbau einer Kompetenz „dialektale Literalität“ als Vermittlungsstoff - für eine in Schleswig-Holstein für die Jahre nach 2018 geplante und in Hamburg seit 2014 bereits umgesetzte Verankerung eines Niederdeutschunterrichts in der Sekundarstufe ist er sogar, orientiert an anderen sprachlichen Fächern, unabdingbar. - Allerdings hätte diese vermittelte dialektale Literalität nur einen mittelbaren Bezug zur mundartliterarischen Tradition und keinen Bezug zu neuen Formen dialektaler Schriftlichkeit in den sozialen Netzwerken. Freiheitlich-kreative Vorstellungen von schriftsprachlichen Ausprägungen müssten zunächst Regelapparaten weichen, bevor die Sprachlerner zur kreativen Sprachanwendung geführt oder zurückgeführt werden können - ein Vorhaben, das sich als zusätzliches Lernziel unbedingt einstellen sollte.

Diese auch utopisch und unter Umständen sogar überflüssig anmutenden Entwicklungen sind mit sprachpolitischer Konsequenz gedacht. Rezente Sprachpolititk fordert eine - jedoch keineswegs neue - Bildungsoption für das Niederdeutsche von der Krippe bis zur Hochschule. ${ }^{17}$ Dieser institutionalisierte Bildungsweg ist ohne Ausprägung auch produktiver literaler Kompentenz in einer niederdeutschen Mundart spätestens ab der Sekundarstufe nicht mehr sinnvoll umsetzbar. Die Grundlagen dieser Kompetenz können zunächst vielfältig sein, denn zahlreiche Felder dialektaler Literalität wurden unterschieden (siehe oben): Die Mundartliteraturgeschichte hat seit dem späten 18. Jahrhundert eine Vielzahl von „Literaturmundarten“ ausgeprägt, die zu ihrem größten Teil jedoch abgeschlossen oder rückläufig sind. In der Fortsetzung treten an ihre Stelle teil-standardisierte, sekundär vermittelte Konzepte dialektaler Literalität zur Bewahrung kultursprachlicher Funktionen, die vornehmlich in der Literatur zu finden sind. Der Kreis der Protagonisten wird kleiner und die schriftlichen Sprachformen dadurch im Zweifelsfall normierter; doch auch dieses Korpus bestehender niederdeutscher Literatur bildet einen gegenwärtigen Lehr-Lern-Stoff und mag eventuell in diesem Rezeptionsumfeld sogar zu einem besonderen Erfolg gelangen, indem die Texte im Unterricht kreativ und kontinuierlich bearbeitet werden.

Es gibt gegenwärtig daneben nur wenige Anwendungsfelder weitgehend geregelter niederdeutscher Schriftlichkeit außerhalb literarischer Texte. Eine kleine Gruppe sprachpolitisch engagierter Sprachaktivisten verwendet niederdeutsche Schriftlichkeit für interne und externe Kommunikation (so z. B. Rundbriefe, E-Mails, Protokolle, sprach-, bildungs- und kulturpolitische Thesenpapiere etc.) in nicht unerheblichem Umfang. Diese Schriftlichkeit ist an der eigenen aktiven Mündlichkeit orientiert, unterwirft sich aber den Regeln eines gruppenintern anerkannten orthographischen Regelwerks für das Niederdeutsche (zum Beispiel des SASSWörterbuchs Kahl/Thies 2013), das gleichzeitig als ein Aushängeschild der eigenen Bewe-

17 Cf. hierzu den Handlungsplan Sprachenpolitik, der 2015 durch den Ministerpräsidenten des Landes Schleswig-Holstein veröffentlicht wurde und Leitlinien und Zielrichtungen der Sprachpolitik des Bundeslandes formuliert: www.schleswig-holstein.de/DE/Fachinhalte/M/minderheiten/Downloads/152906_Handlungsplan_Sprachenpolitik.html [6. 9. 2016]. 
gung gesehen wird. Dieser Gruppe kommt Erfolg zu, da sie in entsprechenden Gremien politischen Einfluss nehmen und durch die dankbar angenommene ehrenamtliche Mitarbeit politische Entscheidungsprozesse auch mittragen und mitgestalten kann. Entsprechende politische Forderungen dieser Interessengruppe sind durch die Europäische Charta der Regional- oder Minderheitensprachen so weitreichend gestützt, dass Umsetzungserfolge nicht ausbleiben.

Aus der Mundartliteratur und den Bemühungen der Sprachaktivisten ergibt sich als neues Profil - auch wenn es bisher kaum herausgestellt wurde - dialektale Schriftlichkeit als institutionell vermittelte Kompetenz im Rahmen mehrjährigen Spracherwerbs. Diese dialektale Schriftlichkeit braucht Standardisierungen und hängt dabei von traditioneller Dialektliteratur und ihrem Konzept einer Imitation standardsprachlicher Schriftlichkeit ab. Der kreative Impuls dialektaler Schriftlichkeit im Netz wird flankiert durch die Vorstellung, dass eine dialektale Schriftlichkeit regelgeleitet erlernbar ist, um damit auch dialektale Mündlichkeit zu sichern. Wenn dieses Konzept einer Sicherung von Mündlichkeit durch den gesteuerten Erwerb von Schriftlichkeit wegen seiner Abweichung von natürlicheren sprachlichen Entwicklungsprozessen auch angreifbar bleibt, so bietet es doch eine entscheidende Profilierung von dialektaler Schriftlichkeit zu Beginn des 21. Jahrhunderts - also zu einem Zeitpunkt, an dem die gesprochenen Dialekte stark rückläufig sind. Eine gewisse Zwangsläufigkeit kommt der Entwicklung auch insofern zu, als dass eine kontinuierlich fortschreitende Sprachvermittlung im institutionellen Rahmen grundsätzlich nicht ohne den Einsatz von Schriftlichkeit gedacht und umgesetzt wird.

Welche Funktion haben somit Übergänge von dialektaler Mündlichkeit in die Schriftlichkeit? Allen gesteuerten Verfahren ist der sprachliche Erhaltungswille gemein; bei den ungesteuerten Verfahren gelten sprachspielerische und sprachpraktische Motive als bestimmend. Im Vorangegangenen wurde der Schwerpunkt auf bewusst gesteuerte Verfahren zur Dialektverschriftlichung gelegt. Verfahrensunterschiede ergeben sich durch die Statusveränderung der Dialekte im gesamtsprachlichen Gefüge: Galt es zu Beginn, einer sprachalltäglich hinlänglich bekannten Sprachform den Weg in die Schriftlichkeit zu bahnen, ist diese Schriftlichkeit inzwischen vielfach die einzige erreichbare Begegnungsmöglichkeit mit einem Dialekt. - Hervorhebung verdienen experimentelle Phasen der Dialektverschriftlichung, die zumindest im Niederdeutschen nur in einem bestimmten sprach- und literarhistorischen Zeitfenster (1960er bis 1980er Jahre) möglich waren (cf. Langhanke 2010). Lohnend bleibt der Blick auf literarische Einzeltexte und deren Entscheidungen zur Lexik, Grammatik und Orthographie - entsprechende Analysen sind in Vorbereitung.

Zudem wird dialektale Literalität didaktisch-methodisch gedacht. Der moderne Fremdsprachunterricht bietet konkrete Ansatzpunkte. Die Methoden des „Storytellings“ und des „StoryPresentations" generieren ausgehend von Schriftlichkeit Mündlichkeit im Unterricht, ${ }^{18}$ indem durch Narration verlebendigte Sprache der Bereitstellung von Wissen und vor allem als Folie für aktives Sprachhandeln dient. Der Ausgangspunkt sind Texte, die auch den Sprachvermittler bei der Vorbereitung stützen. Auf diese Weise wird die literat konservierte Sprache zum Ausgangspunkt neuer Mündlichkeit. Das ist dann bedeutsam, wenn keine intakten Strukturen

18 Cf. zu den fremdsprachdidaktischen Unterrichtsmethoden in Bezug auf den Niederdeutschunterricht Bendixen 2016 und Wormuth 2015. 
von Sprechsprachlichkeit mehr gegeben sind, die sowohl aktive Mündlichkeit als auch die auf ihr aufbauende Schriftlichkeit tragen können.

Der diskutierte Übergang zur Schriftlichkeit bleibt exklusiv und individuell - sowie in den Augen vieler Sprachkritiker überflüssig; aber dennoch ist er für den Erhalt dialektaler Sprachlichkeit konstitutiv, da nur die schriftsprachliche Fixierung eine neue Dimension der Kontinuität und Wertschätzung dialektaler Sprachformen ermöglichen konnte (cf. einschlägig Arendts Diskursanalyse zum 19. Jahrhundert: Arendt 2010; sowie zudem Langhanke 2015b). Literalität ermöglichte neues Prestige (cf. bes. Arendt 2010: 92-100). - Diesen Umstand beweisen sowohl die frühe Geschichte der Dialektologie und der Mundartliteratur als auch die gegenwärtige Verknüpfung dialektaler Schriftlichkeit mit sprachdidaktischen Zielsetzungen. Es kann keinen Erwerb des Niederdeutschen als Fremdsprache ohne Schriftlichkeit geben.

Bei einer Pressevorstellung des Niederdeutsch-Lehrbuches Paul un Emma snackt Plattdü̈tsch (Ashtarany et al. 2015) für die 1. und 2. Klasse mit deutlichen Schriftanteilen wurde im September 2015 betont, dass das Konzept übergreifend sei. Die verwendete Schriftsprache sei normiert, die Aussprache dürfe jedoch landschaftstypisch variieren: Dieses Konzept kann jedoch nur bedingt funktionieren, da es lexikalische, morphologische und auch phonetische Varianz zwischen den Mundarten übersieht, die über Aussprachebesonderheiten hinausgehen. Auf dialektaler Ebene sind die Unterschiede zu groß. Weshalb wird diese Idee dennoch vertreten? Der Schriftsprache wird normierende, einigende Funktion zugesprochen, um das Bildungsprojekt Niederdeutsch zu stützen. Die einfache Aufnahme eines solchen Konzepts kann jedoch nicht erfolgen, wenn die gesamte neuniederdeutsche schriftsprachliche Tradition den anderen Weg der Vielfalt regionaler „Literaturmundarten“ gegangen ist. Zumindest großräumlich ist diese Vielfalt weiterhin anzuerkennen und abzubilden, auch um die jeweiligen schriftsprachlichen Traditionen rezipierbar zu halten. Das genannte Schulbuch ist im zweiten Schritt entsprechend nutzbar, indem die Sprachanteile jeweils für die mundartlichen Großräume des Niederdeutschen ausgetauscht werden - auch dieses mögliche Vorgehen ist Teil seiner auch regional orientierten Konzeption. Ob entsprechenden Lehrbüchern, so ist der Folgeband Paul un Emma II für die Klassenstufen 3 und 4 als Projekt einer an der Universität Flensburg angesiedelten Arbeitsgruppe in Entstehung begriffen, zukünftig ihre eigene normierende Wirkung dennoch zuerkannt werden kann, bleibt kritisch abzuwarten.

\section{$6 \quad$ Fazit}

Die Interpretation eines historischen, im 19. Jahrhundert angesiedelten sprach- und literarhistorischen Diskurses über niederdeutsche Schriftlichkeit wurde vorangehend für eine Perspektivierung der seinerzeit grundgelegten Entwicklungsschritte von niederdeutscher Sprache und Literatur vornehmlich in ihrer schriftlichen Existenzform genutzt; dass neuniederdeutsche Literatur auch eine entscheidende mündliche Tradierung erfährt und erfuhr, konnte an dieser Stelle vernachlässigt bleiben (cf. jedoch Langhanke 2015c) - ragt aber direkt auch in die sprachlichen Vermittlungskontexte, vornehmlich an Schulen und Kindertagesstätten, hinein.

Von der Abbildung kleinräumiger Dialektvarietäten entwickelt sich dialektale Literalität zur Basis einer zunehmend standardisierten Schriftsprache für einen Spracherwerbsunterricht. Der Rückgang gesprochener Dialekte lässt die Entwicklung standardisierter Formen dialektaler Literalität folgerichtig erscheinen, wenn hier auch weniger eine Entwicklung konsequent 
fortgeführt als ein temporärer sprachpolitischer Wille mit konkreten sprachbezogenen Vorschlägen flankiert wird. Ob diese neue Ausprägung teilnormierter dialektaler Schriftlichkeit einen solch durchschlagenden Erfolg auch für die Re-Etablierung dialektaler Mündlichkeit erzielen kann, dass sogar an neue mundartliterarische Formen ausgehend von neuer dialektaler Mündlichkeit zu denken wäre, ist unwahrscheinlich (cf. das Schaubild Langhanke 2015a, 536). Ein Erhalt teilnormierter dialektaler Literalität zur Wahrung einiger schriftsprachlicher Funktionen von Mundarten ist jedoch vorstellbar: An die Stelle lebendiger Gesprochensprachlichkeit mit kreativ anschließenden Verschriftlichungen tritt schriftsprachliche Standardisierung mit gesteuert anschließenden Lernervarietäten: Die Vielfalt der Verschriftlichungsprofile bleibt abhängig von der Lebendigkeit der gesprochenen Dialekte.

Im Juni 1878 formulierte Klaus Groth in einem erkenntnisreichen und daher gern zitierten Vorwort die folgende, sehr weitreichende Überlegung:

Wenn das Plattdeutsche einmal als Volkssprache verschwunden sein wird [...], so wird es doch jetzt in Schriftwerken fortleben.[...] Aber selbst wenn die Liebhaber plattdeutscher Dichtung aussterben, wenn das lebendige Verständnis der Sprache aufhören sollte, so werden die Höchstgebildeten germanischen Stammes im gelehrten Studium zurückgreifen, wieder lernen müssen auf Universitäten und Hochschulen, was ihre Vorfahren gesprochen und gedichtet [...].

(Groth 1878, V)

Das Konzept eines Übergangs niederdeutschen Sprachlebens primär auf eine schriftliche Existenzform hat somit eine lange Tradition, neu ist der Wunsch nach einer durch Sprachvermittlung wieder neu generierten Mündlichkeit.

Es bleibt auffällig, dass die eingangs beschriebenen weiteren, in Teilen noch oder auch erst jüngst produktiven Formen niederdeutscher Schriftlichkeit an dieser Entwicklung nur bedingt Anteil haben. Es sind unterschiedliche Schriftlichkeitsdiskurse über das Niederdeutsche zu unterscheiden, unter denen der mundartliterarische als der historisch und rezent erfolgreichste herauszustellen ist. Während im Falle der Verschriftlichungen in den neuen Medien keine Verbindungen zur mundartliterarischen Tradition neuniederdeutscher Schriftlichkeit herzustellen ist, kann die Schriftlichkeit in der sprachproduktiven Vereinskommunikation durchaus als mundartliterarisch angeregt gelten, da Mundartautoren diese Vereinsgruppierungen durch ihre mehrheitliche Mitwirkung in der Regel bilden und prägen. Die im Beitrag erkennbare Fokussierung der vermittlungsorientierten Verknüpfungen mit niederdeutscher Schriftlichkeit dient einer Vergegenwärtigung der Traditionen, Bedingungen und Möglichkeiten eines solchen ambitionierten Bildungsexperiments für das Niederdeutsche, dessen Erfolge empirischen, evaluativen Verfahren zu unterziehen sein werden. ${ }^{19}$

19 Eine erste Evaluation der 29 schleswig-holsteinischen niederdeutschen Modell-Grundschulen (Erteilung eines durchgängigen Spracherwerbsunterrichts im Niederdeutschen von Klasse 1-4) wurde im Winter 2016/2017 und im Frühjahr 2017 mit Unterstützung der Universität Flensburg und des Instituts für Qualitätssicherung an Schulen Schleswig-Holsteins von Caroline Bruhn und Alexander Radloff durchgeführt, wobei auch ein sprachpolitisches Interesse an den Ergebnissen zu verzeichnen ist. Diese Ergebnisse wurden im Sommer 2017 vorgelegt, können sich jedoch aus methodischen Gründen noch nicht auf individuelle Lernerfolge der Schüler, sondern vornehmlich auf das Lehrerhandeln, auf Methoden, Materialien, konkrete Umsetzungen und Forderungen beziehen. Der materialbezogene Ansatz der Erhebung ist auch für die hier diskutierten Fragestellungen von Interesse, da Aufschlüsse über den Einsatz von schriftlichen Sprachformen in jedem Fall erwartbar sind.

ISSN 1615-3014 


\section{Literaturverzeichnis}

Arendt, Birte (2010): Niederdeutschdiskurse. Spracheinstellungen im Kontext von Laien, Printmedien und Politik. Berlin: Erich Schmidt. (= Philologische Studien und Quellen 224).

Ashtarany, Nicola et al. (2015): Paul un Emma snackt Plattdüütsch. Hamburg: Quickborn.

Batt, Kurt (1958): Untersuchungen zur Auseinandersetzung um Klaus Groth und Fritz Reuter. Unpublished $\mathrm{PhD}$ dissertation, Universität Leipzig.

Batt, Kurt (1967a): Fritz Reuter. Leben und Werk. Bd. 9: Gesammelte Werke und Briefe Fritz Reuters. Rostock: Hinstorff.

Batt, Kurt (1967b): „Die Auseinandersetzung mit Klaus Groth“. In: Batt, Kurt: Fritz Reuter. Leben und Werk. Bd. 9: Gesammelte Werke und Briefe Fritz Reuters. Rostock, Hinstorff: 210-226.

Bendixen, Svenja (2016): Methodische Überlegungen zum Zweitspracherwerb des Niederdeutschen in der Schule. Flensburg: Europa-Universität Flensburg. (=Flensburger Arbeitshefte zur Didaktik der Niederdeutschen Sprache und Literatur 1).

Bichel, Ulf (1973/1974): „Über Urfassung und Spätfassung der plattdeutschen Idyllen von Johann Heinrich Voß und deren Bedeutung für die Mundartliteratur“. Jahresgabe der Klaus-Groth-Gesellschaft 16: 84-97.

Bichel, Inge/Bichel, Ulf/Hartig, Joachim (1994): „Klaus Groth. Eine Bildbiographie“. Jahresgabe der Klaus-Groth-Gesellschaft 36.

Debus, Friedhelm (1996): Von Dünkirchen bis Königsberg. Ansätze und Versuche zur Bildung einer niederdeutschen Einheitssprache. Vorgelegt in der Sitzung vom 20. Oktober 1995. Göttingen: Vandenhoeck \& Ruprecht. (=Berichte aus den Sitzungen der JoachimJungius-Gesellschaft der Wissenschaften e. V. Hamburg 14/2).

Denkler, Markus (2006): Sterbfallinventare. Text- und variablenlinguistische Untersuchungen zum Schreibsprachenwechsel in Westfalen (1500-1800). Köln/Weimar/Wien: Böhlau. (= Niederdeutsche Studien 52).

Elmentaler, Michael (2009a): „Modernes Niedersächsisch - Dialektwandel im nordniederdeutschen Raum“. In: Lenz, Alexandra N./Gooskens, Charlotte/Reker, Siemon (eds.): Low Saxon Dialects across Borders - Niedersächsische Dialekte über Grenzen hinweg. Stuttgart, Franz Steiner: 339-365. (=Zeitschrift für Dialektologie und Linguistik. Beihefte 138).

Elmentaler, Michael (2009b): „Hochdeutsch und Platt - zwei ungleiche Nachbarn“. In: Elmentaler, Michael (ed.): Deutsch und seine Nachbarn. Frankfurt a. M., Lang: 31-45. (= Kieler Forschungen zur Sprachwissenschaft 1).

Elmentaler, Michael/Borchert, Felix (2012): „Niederdeutsche Syntax im Spannungsfeld von Kodex und Sprachpraxis“. In: Langhanke, Robert et al. (eds.): Niederdeutsche Syntax. Hildesheim/Zürich/New York, Georg Olms: 101-135. (= Germanistische Linguistik 220).

Elmentaler, Michael (2015): „Dialektaler Wandel in Schleswig-Holstein. Die wechselvolle Geschichte von et und dat". In: Langhanke, Robert (ed.): Sprache, Literatur, Raum. Festgabe für Willy Diercks. Bielefeld, Verlag für Regionalgeschichte: 301-351.

Eschenhagen, Heinrich (1860): Zur plattdeutschen Sprache und deren neue Literaturbewegung. Berlin: E. Schotte.

Fredsted, Elin (2015): „Mündlichkeit und Schriftlichkeit“. In: Fredsted, Elin/Langhanke, Robert/Westergaard, Astrid (eds.): Modernisierung in kleinen und regionalen Sprachen. Hildesheim/Zürich/New York, Georg Olms: 1-31. (= Kleine und regionale Sprachen 1). 
Goltz, Reinhard (1996): „Versteiht mi denn keeneen? Plattdeutsche Lyrik zwischen Mundart und Standardsprache“. In: Dohnke, Kay/Schröder, Ingrid/Spiekermann, Gerd (eds.): Vun Böker un Minschen. Festschrift für Friedrich W. Michelsen. Hamburg, Quickborn: 74-88.

Goltz, Reinhard (1998): „Niederdeutsche Literatur - ein enges Feld? Neue niederdeutsche Texte zwischen Originalität und Sprachpflege“. Jahresgabe der Klaus-Groth-Gesellschaft 40: $57-108$.

Goltz, Reinhard (2013): „Wi schrievt op Platt - Schreiben und Lesen im NiederdeutschUnterricht". In: Ehlers, Christiane/Goltz, Reinhard/Henschen, Walter (eds.): Auf dem Stundenplan: Plattdeutsch. Leer, Schuster: 32-33. (= Schriften des Instituts für niederdeutsche Sprache 45).

Goltz, Reinhard (2015a): „Seggt de een to den annern ... Wat en Dialog mit den Text maakt. En Lex ut de Schrievwarksteed“. In: Langhanke, Robert (ed.): Sprache, Literatur, Raum. Festgabe für Willy Diercks. Bielefeld, Verlag für Regionalgeschichte: 611-624.

Goltz, Reinhard (2015b): „Niederdeutsch im Bildungswesen in den norddeutschen Bundesländern - ein Vergleich“. In: Nath, Cornelia (ed.): Bildungs- und Integrationschancen durch Niederdeutsch. Dokumentation der Oll' Mai-Veranstaltung am 10. Mai 2014 in der Nicolaikirche in Wittmund. Aurich, Verlag der ostfriesischen Landschaft: 17-38.

Groth, Klaus (1852/1938): „Klaus Groth an Ernst Friedrich Christan Griebel. Landkirchen, 11. September 1852 / 1. Oktober 1852.“ In: Pauls, Volquart (ed.): Um den Quickborn. Briefwechsel zwischen Klaus Groth und Karl Müllenhoff. Neumünster, Karl Wachholtz: 298-305. (= Sprache und Schriftum. Neue Folge der Forschungen, Reihe B 1).

Groth, Klaus (1852/1853): Quickborn. Volksleben in plattdeutschen Gedichten ditmarscher Mundart nebst Glossar. Mit einem Vor- und Fürwort von Oberconsistorialrath Pastor Dr. Harms in Kiel. Hamburg: Perthes, Besser und Mauke.

Groth, Klaus (1858/1981): „Briefe über Hochdeutsch und Plattdeutsch“. In: Braak, Ivo/Mehlem, Richard (eds.): Klaus Groth: Sämtliche Werke. Bd. 6: Über Sprache und Dichtung. Heide, Boyens: 67-137.

Groth, Klaus (1864/2005): „Die patriotische Wirksamkeit eines schleswig-holsteinischen Privatmannes“. In: Bichel, Ulf/Goltz, Reinhard (eds.): Klaus Groth: Memoiren. Heide, Boyens: 59-88.

Groth, Klaus (1865-1866/1981): „Erhaltung der Volkssprache“. In: Braak, Ivo/Mehlem, Richard (eds.): Klaus Groth: Sämtliche Werke. Bd. 6: Über Sprache und Dichtung. Heide, Boyens: 139-173.

[Groth, Klaus] (1876): Einige praktische Vorschläge zu einer einheitlichen plattdeutschen Schreibweise für plattdeutsche Schriftsteller. Leipzig: Eigenverlag/Privatdruck. [anonym erschienen, vom Autor des vorliegeneden Beitrags aus inhaltlichen Gründen Groth zugeordnet].

Groth, Klaus (1872-1873/1981): „Über Mundart und mundartige Dichtung“. In: Braak, Ivo/Mehlem, Richard (eds.): Klaus Groth: Sämtliche Werke. Bd. 6: Über Sprache und Dichtung. Heide, Boyens: 175-245.

Groth, Klaus (1878): „Vorwort“. In: Giese, Franz (ed.): Frans Essink. Sin Leben un Driben as olt Münstersch Kind. 3. Auflage. Braunschweig, Harald Bruhn: V-VII.

Groth, Klaus (1885/1981): „Eine plattdeutsche Bibelübersetzung“. In: Braak, Ivo/Mehlem, Richard (eds.): Klaus Groth: Sämtliche Werke. Bd. 6: Über Sprache und Dichtung. Heide, Boyens: $311-322$. 
Groth, Klaus (1889/1981): „Wie mein Quickborn entstand“. In: Braak, Ivo/Mehlem, Richard (eds.): Klaus Groth: Sämtliche Werke. Bd. 6: Über Sprache und Dichtung. Heide, Boyens: $35-65$.

Haas, Walter (1983): „Dialekt als Sprache literarischer Werke“. In: Besch, Werner et al. (eds.): Dialektologie. Ein Handbuch zur deutschen und allgemeinen Dialektforschung. Berlin/New York, de Gruyter: 1637-1651. (= Handbücher zur Sprach- und Kommunikationswissenschaft 1.2).

Herrmann, Leonhard/Siebenhaar, Beat (2015): „Fiktive Sprachen. Wie der Dialekt in die Literatur kommt - ein dialektologisch-literaturwissenschaftliches Lehr- und Forschungsprojekt“". In: Bär, Jochen A./Mende, Jana-Katharina/Steen, Pamela (eds.): Literaturlinguistikphilologische Brückenschläge. Frankfurt a. M., Lang: 47-73. (=Littera. Studien zu Sprache und Literatur / Studies in Language and Literature 6).

Hinsch, Gerhard (1956): Probleme und Geschichte der neuniederdeutschen Schreibweisen im Nordniedersächsischen. Unpublished PhD dissertation, Universität Hamburg.

Hinsch, Gerhard (1983): „Schreibung des Niederdeutschen“. In: Cordes, Gerhard/Möhn, Dieter (eds.): Handbuch zur niederdeutschen Sprach- und Literaturwissenschaft. Berlin, Erich Schmidt: 182-205.

Holsten, Christian (1964): „Christian Holsten“. In: Keseling, Gisbert/Mews, Hans-Joachim (eds.): Gespräche mit plattdeutschen Autoren. Herausgeben von den Mitarbeitern des Niedersächsischen Wörterbuchs. Neumünster, Karl Wachholtz: 44-50.

Hückstädt, Arnold (ed.) (2009a): Fritz Reuter. Briefe. Bd. 1.: 1827-1860. Rostock: Hinstorff. Hückstädt, Arnold (ed.) (2009b): Fritz Reuter. Briefe. Bd. 2.: 1861-1866. Rostock: Hinstorff. Hundt, Markus (2000): „Spracharbeit“ im 17. Jahrhundert. Studien zu Georg Philipp Harsdörffer, Justus Georg Schottelius und Christian Gueintz. Berlin/New York: de Gruyter. (= Studia Linguistica Germanica 57).

Jaeger, Monika (1964): Theorien der Mundartdichtung. Studien zu Anspruch und Funktion. Tübingen: Tübinger Vereinigung für Volkskunde. (=Volksleben. Untersuchungen des Ludwig-Uhland-Instituts der Universität Tübingen i. A. der Tübinger Vereinigung für Volkskunde 3).

Jahn, Moritz (1964): „Moritz Jahn“. In: Keseling, Gisbert/Mews, Hans-Joachim (eds.): Gespräche mit plattdeutschen Autoren. Herausgeben von den Mitarbeitern des Niedersächsischen Wörterbuchs. Neumünster, Karl Wachholtz: 50-57.

Jørgensen, Peter (1934): Die dithmarsische Mundart von Klaus Groths „Quickborn “. Lautlehre, Formenlehre, Glossar. Kopenhagen: Levin und Munsgaard.

Kahl, Heinrich/Thies, Heinrich (2013): Der neue SASS. Plattdeutsches Wörterbuch. Plattdeutsch-Hochdeutsch. Hochdeutsch-Plattdeutsch. Plattdeutsche Rechtschreibung. Herausgegeben von der Fehrs-Gilde e. V. 7. Auflage. Neumünster: Wachholtz.

Kellner, Birgit (2002): Zwischen Anlehnung und Abgrenzung. Orthographische Vereinheitlichung als Problem im Niederdeutschen. Heidelberg: Winter. (= Sprachgeschichte 7).

Kellner, Birgit (2005): „Wieviel Standard benötigt das Niederdeutsche? Überlegungen zur Kodifizierung und Normierung einer, weniger gebrauchten Sprache““. Korrespondenzblatt des Vereins für niederdeutsche Sprachforschung 112: 17-27.

Kleiner, Stefan (2003): Geschriebener Dialekt in Bayerisch-Schwaben. Ein Vergleich indirekt erhobener dialektaler Laienschreibungen mit ihren lautschriftlichen Entsprechungen. Berlin/New York: de Gruyter. (= Phonai 48). 
Kloss, Hinz (1987): „Abstandsprache und Ausbausprache“. In: Ammon, Ulrich/Dittmar, Norbert/Mattheier, Klaus J. (eds.): Soziolinguistik. Ein internationales Handbuch zur Wissenschaft von Sprache und Gesellschaft. Berlin/New York, de Gruyter: 302-308. (= Handbücher zur Sprach- und Kommunikationswissenschaft 3.1).

Knoop, Ulrich (1982): „Das Interesse an den Mundarten und die Grundlegung der Dialektologie“. In: Besch, Werner et al. (eds.): Dialektologie. Ein Handbuch zur deutschen und allgemeinen Dialektforschung. Berlin/New York, de Gruyter: 38-92. (=Handbücher zur Sprach- und Kommunikationswissenschaft 1.2).

Langhanke, Robert (2009): „Dichtung und Dialektologie. Verbindungen zwischen westfälischer Mundartliteratur und Mundartforschung im 19. Jahrhundert“. Augustin WibbeltGesellschaft. Jahrbuch 25: 7-43.

Langhanke, Robert (2010): „Neuniederdeutsche Literatur: Über Beginn und nahenden Abschluss einer überschaubaren Literaturtradition“. In: Munske, Horst Haider (ed.): Dialektliteratur heute - regional und international. Forschungskolloquium am Interdisziplinären Zentrum für Dialektforschung an der Friedrich-Alexander-Universität ErlangenNürnberg, 19.11.2009-20.11.2009. Erlangen, Interdisziplinäres Zentrum für Dialektforschung: 4-40. www.dialektforschung.phil.uni-erlangen.de/publikationen/dialektliteraturheute.shtml [6.09.2016)].

Langhanke, Robert (2011a): „Von dichtenden Dialektologen und dialektologischer Dichtung: Mundartdichtung als Textkorpus früher Dialektologie und ihr heutiger Quellenwert“. In: Christen, Helen/Patocka, Franz/Ziegler, Evelyn (eds.): Struktur, Verwendung und Wahrnehmung von Dialekt. Beiträge zum 3. Kongress der Internationalen Gesellschaft für Dialektologie des Deutschen (IGDD), Zürich, 7.-9. September 2009. Wien, Praesens: 99-126.

Langhanke, Robert (2011b): „Oesterhaus versus Wenker. Überlegungen zum Quellenwert von Mundartdichtung“". Augustin-Wibbelt-Gesellschaft. Jahrbuch 27: 67-96.

Langhanke, Robert (2012a): „Der Arzt von der Bega und die lippische Mundartdichtung: Zum 75. Todestag des Mundartdichters Korl Biegemann und Medizinalrates Ulrich Volkhausen“. Augustin Wibbelt-Gesellschaft. Jahrbuch 28: 57-85.

Langhanke, Robert (ed.) (2012b): Robert Peters. Mittelniederdeutsche Studien. Gesammelte Schriften 1974 bis 2003. Bielefeld: Verlag für Regionalgeschichte.

Langhanke, Robert (2013): „Zweit- und Lernersprache Niederdeutsch - Aufgaben und Perspektiven einer renovatio linguae saxonicae“. In: Zybatow, Tatjana/Harendarski, Ulf (eds.): Sprechen, Denken und Empfinden. Berlin, Lit: 297-312. (= Germanistik 43).

Langhanke, Robert (2015a): „Zur literarischen Wiedersichtbarmachung des Niederdeutschen im 19. Jahrhundert. Konzepte und Konflikte der niederdeutschen Reliterarisierung“. In: Langhanke, Robert (ed.): Sprache, Literatur, Raum. Festgabe für Willy Diercks. Bielefeld, Verlag für Regionalgeschichte: 479-536.

Langhanke, Robert (2015b): „Zur Bedeutung Klaus Groths für die niederdeutsche Sprachgeschichte“. In: Hundt, Markus/Lasch, Alexander (eds.): Deutsch im Norden. Varietäten des norddeutschen Raumes. Berlin/Boston, de Gruyter: 319-349. (= Jahrbuch für Germanistische Sprachgeschichte 6).

Langhanke, Robert (2015c): „Zurück zur Sprache über die Literatur - die subliterarischen Suchen des Sauerländers Peter Bürger oder: Was ist und wo finde ich niederdeutsche Literatur?" Bevensen-Jahrbuch 67: 170-198. 
Langhanke, Robert (2016): „Literalität und regionale Sprache. Herausforderungen neuniederdeutscher Schriftlichkeit in historischer Perspektivierung und die Positionen Groths und Reuters“. In: Januschek, Franz (ed.): Transkulturelle Perspektiven auf mehrsprachige Regionen. Fes und Flensburg im Dialog. Hildesheim/Zürich/New York, Georg Olms: 92131. (= Kleine und regionale Sprachen 2).

Langhanke, Robert (2017/im Druck): „Rezente und historische Standardisierungsdiskurse über das Niederdeutsche“. In: Ahlers, Timo et al. (eds.): Deutsche Dialekte in Europa. Perspektiven auf Variation, Wandel und Übergänge. Hildesheim/Zürich/New York, Georg Olms: 229-260. (= Kleine und regionale Sprachen 3).

Lindow, Wolfgang et al. (1998): Niederdeutsche Grammatik. Leer: Schuster. (= Schriften des Instituts für niederdeutsche Sprache. Reihe: Dokumentation 20).

Maas, Utz (1989): „Orthographische Alterität. Über literarische Mundartgraphien“. In: Heimann, Sabine/Lerchner, Gotthard/Müller, Ulrich (eds.): Soziokulturelle Kontexte der Sprach- und Literaturentwicklung. Festschrift für Rudolf Große zum 65. Geburtstag. Stuttgart, Heinz: 339-359. (= Stuttgarter Arbeiten zur Germanistik 231).

Menke, Hubertus (1998): „Niederdeutsch: Eigenständige Sprache oder Varietät einer Sprache?" In: Schmitsdorf, Eva/Hartl, Nina/Meurer, Barbara (eds.): Lingua Germanica. Studien zur deutschen Philologie. Jochen Splett zum 60. Geburtstag. Münster etc., Waxmann: 171-184.

Menke, Hubertus (2000): „Niederdeutsche Literatur“. In: Fricke, Harald et al. (ed.): Reallexikon der deutschen Literaturwissenschaft. Bd. 2. Berlin/New York, de Gruyter: 714-718.

Menke, Hubertus (2001): „Een’ Spraak is man bloots een Dialekt, de sik to Wehr setten kann. Nachlese zur Diskussion um die Europäische Sprachenschutzcharta“. In: Föllner, Ursula (ed.): Niederdeutsch. Sprache und Literatur der Region. Frankfurt a. M., Lang: 9-33. (=Literatur -Sprache-Region. Beiträge zur Kulturgeographie 5).

Meyer, Gustav Friedrich (1923/1983): Unsere platteutsche Muttersprache. Beiträge zu ihrer Geschichte und zu ihrem Wesen. Bearbeitet und neu herausgegeben von Ulf Bichel. St. Peter-Ording: Lühr und Dircks.

Mihm, Arend (2007): Sprachwandel im Spiegel der Schriftlichkeit. Studien zum Zeugniswert der historischen Schreibsprachen des 11. bis 17. Jahrhunderts. Frankfurt a. M: Lang.

Möhn, Dieter (1972): „Zur Problematik der niederdeutschen Literatursprache“. Jahresgabe der Klaus-Groth-Gesellschaft 14: 85-103.

Peters, Robert (1995/2012): „Die angebliche Geltung der sog. mittelniederdeutschen Schriftsprache in Westfalen. Zur Geschichte eines Mythos“. In: Langhanke, Robert (ed.): Robert Peters. Mittelniederdeutsche Studien. Gesammelte Schriften 1974 bis 2003. Bielefeld, Verlag für Regionalgeschichte: 323-341.

Peters, Robert (1998/2012): „Zur Sprachgeschichte des niederdeutschen Raumes“. In: Langhanke, Robert (ed.): Robert Peters. Mittelniederdeutsche Studien. Gesammelte Schriften 1974 bis 2003. Bielefeld, Verlag für Regionalgeschichte: 443-461.

Peters, Robert (2017/im Druck): Atlas spätmittelalterlicher Schreibsprachen des niederdeutschen Altlandes und angrenzender Gebiete (ASnA). In Zusammenarbeit mit Christian Fischer und Norbert Nagel. Bd. 1: Einleitung, Karten. Bd. 2: Verzeichnis der Belegtypen. Bd. 3: Verzeichnis der Schreibformen und der Textzeugen (Ortspunktdokumentation). Berlin/Boston: de Gruyter. 
Polenz, Peter von (2013): Deutsche Sprachgeschichte vom Spätmittelalter bis zur Gegenwart. Bd. 3: 17. und 18. Jahrhundert. 2. Auflage. Berlin/Boston: de Gruyter.

Reershemius, Gertrud (2015a): „Ein Tag mit Plattdeutsch. Mehrsprachige Praktiken zwischen Tradition und Globalisierung“. In: Langhanke, Robert (ed.): Sprache, Literatur, Raum. Festgabe für Willy Diercks. Bielefeld, Verlag für Regionalgeschichte: 393-409.

Reershemius, Gertrud (2015b): „Kleinere, regionale und Minderheitensprachen im Web 2.0 am Beispiel des Niederdeutschen“. In: Fredsted, Elin/Langhanke, Robert/Westergaard, Astrid (eds.): Modernisierung in kleinen und regionalen Sprachen. Hildesheim/Zürich/New York, Georg Olms: 53-83. (= Kleine und regionale Sprachen 1).

Reichmann, Oskar (1990): „Sprache ohne Leitvarietät vs. Sprache mit Leitvarietät: ein Schlüssel für die nachmittelalterliche Geschichte des Deutschen?“ In: Besch, Werner (ed.): Deutsche Sprachgeschichte. Grundlagen, Methoden, Perspektiven. Festschrift für Johannes Erben zum 65. Geburtstag. Frankfurt a. M., Lang: 141-158.

Reuter, Fritz (1859/1966-1967). „Vorrede zur vierten Auflage“. In: Batt, Kurt (ed.): Fritz Reuter. Gesammelte Werke und Briefe. Bd. 2: Läuschen un Rimels. Erste Folge. Läuschen un Rimels. Neue Folge. Polterabend-Gedichte in hochdeutscher und niederdeutscher Mundart. Rostock, Hinstorff: 7-203.

Reuter, Fritz (1862-1864): Ut mine Stromtid. Bd. 1-3. Rostock: Hinstorff. (= Olle Kamellen $3-5)$.

Rohdenburg, Günter (1991): „Funktionale Unterscheidung bei konkurrierenden Formen des Präteritums von doon ,tun“ im Niederdeutschen“. Niederdeutsches Jahrbuch 114: 164-174.

Rohdenburg, Günter (2002): „Die Umschreibung finiter Verbformen mit ,doon“ ,tun“ und die Frikativierung stammauslautender Plosive in nordniederdeutschen Mundarten". NorthWestern European language evolution (NOWELE) 40: 85-104.

Rohse, Eberhard et al. (eds.) (2011): August Hinrichs und Moritz Jahn. Ein literaturwissenschafticher Vergleich 1870 bis 1970. Frankfurt a. M: Lang. (= Literatur - Sprache - Region. Studien zur Kulturgeographie 8).

Schmidt, Jürgen Erich/Herrgen, Joachim (2011): Theorie der Sprachdynamik. Eine Einführung in die moderne Regionalsprachenforschung. Berlin: Erich Schmidt. (=Grundlagen der Germanistik 49).

Stellmacher, Dieter (1978): „Haben die Niederdeutschen noch eine Literatursprache?“ Jahresgabe der Klaus-Groth-Gesellschaft 20: 85-96.

Stellmacher, Dieter (1996): „Niederdeutsch“. In: Hinderling, Robert/Eichinger, Ludwig Maximilian (eds.): Handbuch der mitteleuropäischen Sprachminderheiten. Tübingen, Narr: 497-505.

Stellmacher, Dieter (ed.) (1986): Studien zu Moritz Jahn. Ergebnisse des wissenschaftlichen Kolloquiums aus Anlaß des 100. Geburtstages des Dichters, Göttingen, den 10. 10. 1984. Rinteln: Bösendahl. (= Name und Wort. Göttinger Arbeiten zur niederdeutschen Philologie 8).

Stiftung F.V.S. zu Hamburg (ed.) (1989): Verleihung des Fritz-Reuter-Preises 1989 an Frau Waltrud Bruhn, Glückstadt, am 7. Juli 1989 in Glückstadt. Hamburg: Verlag der Stiftung F.V.S. zu Hamburg.

Thies, Heinrich (2011): SASS. Plattdeutsche Grammatik. Formen und Funktionen. 2. Auflage. Neumünster: Wachholtz. 
Tophinke, Doris (2008): „Regional schreiben: Weblogs zwischen Orthographie und Phonographie“. In: Christen, Helen/Ziegler, Evelyn (eds.): Sprechen, Schreiben, Hören. Zur Produktion und Perzeption von Dialekt und Standardsprache zu Beginn des 21. Jahrhunderts. Beiträge zum 2. Kongress der Internationalen Gesellschaft für Dialektologie des Deutschen, Wien, 20.-23. September 2006. Wien, Praesens: 153-180.

Wilcken, Viola (2015): Historische Umgangssprachen zwischen Sprachwirklichkeit und literarischer Gestaltung. Formen, Funktionen und Entwicklungslinien des ,,Missingsch“. Hildesheim/Zürich/New York: Georg Olms. (= Deutsche Dialektgeographie/Sprachvariation in Norddeutschland 121).

Wirrer, Jan (2014): „Reviewed Work: ,Niederdeutsche Syntax‘. by Robert Langhanke, Kristian Berg, Michael Elmentaler, Jörg Peters“. Zeitschrift für Dialektologie und Linguistik 81/2: 220-225.

Wormuth, Inga (2015): Neue Methoden für den Niederdeutschunterricht - Impulse aus der Deutsch- und Fremdsprachendidaktik. Unpublished master's thesis, Universität Flensburg. (Publikation in Vorbereitung).

Wyss, Eva/Ziegler, Evelyn (2008): „Dialekt in der privaten Schriftlichkeit von Zürcher Jugendlichen“. In: Christen, Helen/Ziegler, Evelyn (eds.): Sprechen, Schreiben, Hören. Zur Produktion und Perzeption von Dialekt und Standardsprache zu Beginn des 21. Jahrhunderts. Beiträge zum 2. Kongress der Internationalen Gesellschaft für Dialektologie des Deutschen, Wien, 20.-23. September 2006. Wien, Praesens: 131-151. 